\title{
The temporal and spatial evolution of the starburst in ESO 338-IG04 as probed by its star clusters ${ }^{\star}$
}

\author{
G. Östlin ${ }^{1}$, E. Zackrisson², N. Bergvall ${ }^{2}$, and J. Rönnback ${ }^{2}$ \\ 1 Stockholm Observatory, 10691 Stockholm, Sweden \\ 2 Uppsala University, Dept. of Astronomy and Space Physics, Box 515, 75120 Uppsala, Sweden
}

Received 8 November 2002 / Accepted 20 June 2003

\begin{abstract}
ESO 338-IG04, also known as Tololo 1924-416, is a well known luminous blue compact galaxy in the local universe. Images obtained with the Hubble Space Telescope (HST) have shown that the central starburst region is composed of numerous bright point sources - young star clusters, surrounded by a population of old and intermediate age globular clusters. In this paper we use Ultra-Violet (UV) and optical HST photometry in five bands, and an extensive set of spectral evolutionary synthesis scenarios to investigate the age and masses of 124 star clusters. The very small reddening makes ESO 338-IG04 an excellent laboratory for studying the formation of such objects. We find that a careful treatment of the nebular emission component is crucial when modelling the broad-band colours of young starburst regions. We have used the star clusters to trace the temporal and spatial evolution of the starburst, and to put constraints on the star formation activity over a cosmological time-scale. The present starburst has been active for about $40 \mathrm{Myr}$ and shows evidence for propagating star formation and structures triggered by galactic winds. A standard Salpeter initial mass function (IMF) extending up to $120 M_{\odot}$ provides the best fit to the data, although a flatter IMF cannot be excluded. The compact star clusters provide 30-40\% of the UV luminosity and star formation activity. We find no evidence for dust obscuration even among the youngest $(<1 \mathrm{Myr})$ clusters, and we propose that this may be related to a short time-scale for destruction of dusty molecular clouds. Over a longer time-scale, we find evidence for previous cluster formation epochs - notably one a couple of Gyr ago. The fraction of the galaxy's stellar mass contained in compact star clusters is found to be several percent, which is an unusually high value. The intermediate age clusters show a flattened space distribution which agrees with the isophotal shape of the galaxy, whereas the oldest clusters seem to have a spherical distribution indicating that they formed prior to the rest of the galaxy.
\end{abstract}

Key words. galaxies: starburst - galaxies: star clusters - galaxies: dwarf - galaxies: individual: ESO 338-IG04

\section{Introduction}

According to the hierarchical paradigm for structure formation, small structures form first and massive galaxies are successively built up by mergers of smaller units. Blue Compact Galaxies (BCGs) are galaxies with lower mass and metallicity than typical $L^{\star}$ galaxies. Their spectra often show a blue continuum and strong emission lines, indicative of low chemical abundances and active star formation. These properties once led to the idea that BCGs were young systems presently forming their first generation of stars (Searle \& Sargent 1972). Today it is evident that the majority of BCGs are not young (see Kunth \& Östlin 2000 for a review), but rather experiencing a phase of increased star formation activity, ranging from moderate enhancements (Schulte-Ladbeck et al. 2001;

Send offprint requests to: $\mathrm{G}$. Östlin,

e-mail: ostlin@astro.su.se

* Based on observations with the NASA/ESA Hubble Space Telescope, obtained at the Space Telescope Science Institute, which is operated by the association of Universities for Research in Astronomy, Inc., under NASA contract NAS5-26555.
Hopkins et al. 2002) to true starbursts, with short gas consumption time-scales (Östlin et al. 2001). Understanding the reasons for this lies at the heart of the quest to understand the role of BCGs in the cosmic galaxy evolution framework.

Observations of e.g. the Hubble Deep Field (Williams et al. 1996), show that luminous compact blue galaxies are common at higher redshifts (Guzman et al. 1997). Moreover, Ly $\alpha$ emitters at redshift $z=2.4$ have lower continuum luminosity and smaller sizes than expected for young $L^{\star}$ galaxies (Pascarelle et al. 1998). These may be dwarf galaxies, about to build up larger structures by mergers. Several investigations (e.g. Lilly et al. 1996; Madau et al. 1996) show that the cosmic star formation rate (SFR) was much higher at $z \geq 1$, and the same applies to the merger frequency although mergers appear to have played an important role also at $z<1$ (e.g. Le Fèvre et al. 2000). Based on kinematics, morphology and photometry, Östlin et al. (2001) suggest that mergers may be the main mechanism responsible for starbursts in luminous BCGs (LBCGs) in the local universe. This is also supported by the enigmatic under-abundance of the ISM metallicity compared to the stellar population found by Bergvall \& Östlin (2002). Hence, LBCGs 
offer an opportunity to study hierarchical buildup of galaxies in detail.

Globular clusters (GCs) include the oldest known stellar systems in the local universe. Old Galactic halo GCs are around $15 \mathrm{Gyr}$ old and typically have metallicities $[\mathrm{Fe} / \mathrm{H}]=-1.5$ (Ashman \& Zepf 1998). High resolution imaging, notably with the Hubble Space Telescope (HST) of merging galaxies in the local universe have shown that luminous compact star clusters (often referred to as super star clusters) with properties consistent with young GCs are formed in such events (see Whitmore 2002 for a recent review). This can explain the existence of intermediate age (a few Gyr) metal-rich GC populations found in many local elliptical galaxies (see Ashman \& Zepf 1998 for a review). Hence, GC formation was not confined to the high $z$ universe, but is still going on but at a slower pace. Also LBCGs are sites of young compact star clusters (Östlin 2000). However, the ISM abundances $([\mathrm{O} / \mathrm{H}] \approx-1)$ are lower than those in giant mergers, and similar (if $[\mathrm{Fe} / \mathrm{O}] \approx-0.5$, as is common in metal-poor environments, see e.g. Kunth \& Östlin 2000) to the metallicity of old GCs. The first generation of GCs in the universe may have formed in the processes of merging sub-galactic clumps, similar to what is going on in some local LBCGs.

ESO 338-IG04 is a well known luminous $\left(M_{V}=-19.3\right)$ BCG, first studied in detail by Bergvall (1985). Images obtained with the Wide Field Planetary Camera 2 (WFPC2) on board the HST found numerous compact star clusters associated with ESO 338-IG04 (Östlin et al. 1998, hereafter ÖBR98). In addition UV observations with the Faint Object Camera (FOC) on board HST, had already shown that the centre of ESO 338-IG04 hosted a population of UV-bright star clusters, just like many other starbursts (Meurer et al. 1995). Further analysis showed that the star clusters in ESO 338-IG04 spanned ages from a few Myr to more than $10 \mathrm{Gyr}$ and that they had just the properties expected for young and old GCs (ÖBR98). The galaxy is a true starburst in the sense that the time-scales for gas consumption and build-up of the observed stellar mass with the current SFR both are $\sim 1$ Gyr, i.e. much shorter than the age of the universe (Östlin et al. 2001).

In the present paper, we will make a refined analysis of 124 star clusters in ESO 338-IG04 using additional UV data and a new set of models. We use the star clusters and their ageand space-distributions to track the evolution of the star formation in the galaxy over a cosmological time scale.

\section{Observational data}

ESO 338-IG04 was observed with HST/WFPC2 with the galaxy centred on the Planetary Camera (PC) aperture (ÖBR98). The photometry in the F336W, F439W, F555W and F814W bands (hereafter referred to as: $u, b, v, i$ ) is described in ÖBR98. In addition we use photometry in the F218W filter which is very valuable for constraining the physical parameters and the extinction of young objects. The F218W photometry (hereafter $x$ ) has been performed in the same way as for the other passbands, and has been corrected for the same amount of Galactic extinction: $E(B-V)_{\mathrm{G}}=0.09$ (Burstein $\&$ Heiles 1982). We have also corrected the F218W data for a
“UV-contamination” of -0.1276 mag (Biretta et al. 2002). All photometry in this paper is in the VEGAMAG system (Biretta et al. 2002)

Figure 1 shows an RGB true colour composite of the $i, v$ and $b$ images. The $v$ filter (F555W) has significant transmission at $\mathrm{H} \beta$, [O III $]_{\lambda 4959,5007}$ and even $\mathrm{H} \alpha$. Consequently, several nebulous emission-line regions are seen in green in Fig. 1. It is obvious that this galaxy presents a large number of compact star cluster candidates. In ÖBR98 we showed that the vast majority of these are indeed star clusters, physically associated with ESO 338-IG04 (i.e. not foreground stars, background galaxies or individual supergiants in ESO 338-IG04). Their sizes are compatible with globular clusters, although a few of the younger sources are slightly more extended and may represent unbound systems. In Fig. 2 we show an RGB composite of the central region using the $i, v$ and $x$ filters. The variety of colours evident from Figs. 1 and 2: a central population with bright blue clusters surrounded by a population of fainter red ones, directly suggests that the clusters have different age or reddening.

Figure 3 shows the F218W magnitude $\left(m_{x}\right)$ vs. the photometric uncertainties $\left(\sigma_{x}\right)$, and vs. $M_{v}$, the absolute $v$ magnitudes for the same distance as used in ÖBR98 (37.5 Mpc, or $m-M=32.87)^{1}$. From the catalogue in ÖBR98, a total of 78 objects with $m_{x} \leq 22$ were detected. However, cosmic ray contamination is worse (due to longer exposure time, $1800 \mathrm{~s}$ split into two sub-exposures) than in the other filters, and some spurious detections had to be removed by hand. In total, 49 objects have $\sigma_{x} \leq 0.5$ and 21 objects have $\sigma_{x} \leq 0.2$. This is comparable to Meurer et al. (1995) who detected 14 compact sources with the pre-refurbishment HST/FOC and the F220W filter having uncertainties $\sigma_{\mathrm{F} 220 \mathrm{~W}} \leq 0.17$. The photometric agreement is fair when comparing sources for which Meurer et al. (1995) determined the flux from radial profiles. From Fig. 3 it is evident that the sources detected in F $218 \mathrm{~W}$ have very blue $x-v$ colours, with a median around -2 , a combined effect of low age, metallicity and extinction of the UV detected clusters.

Figure 4 shows the $x-u$ vs. $b-v$ two-colour diagram for objects with accurate photometry, together with two typical models used (see below for details on these). During the first 10 Myr the $x-u$ colour is nearly constant making it an efficient probe of the reddening for young objects. On the other hand, $b-v$ evolves strongly, mainly due to the rapid decrease in the contribution from emission lines to the $v$ (F555W) filter. That the objects scatter close to the tracks demonstrate that the extinction is very small. It is also clear that it is only for objects younger than a few times 10 Myr that we have reliable F218W data.

In addition to the clusters in Fig. 1, which are found in the unvignetted field of view (FOV) of the PC, a blue tail of the galaxy (see Bergvall \& Östlin 2002) extends into the WF4 aperture (downwards in the orientation of Fig. 1), where another dozen compact objects are found. This is near the border between the chips, and we do not regard the photometry of these objects reliable. Some objects appear extended,

1 The distance is based on a Hubble parameter of $H_{0}=$ $75 \mathrm{~km} \mathrm{~s}^{-1} \mathrm{Mpc}^{-1}$, which is assumed throughout this paper. 


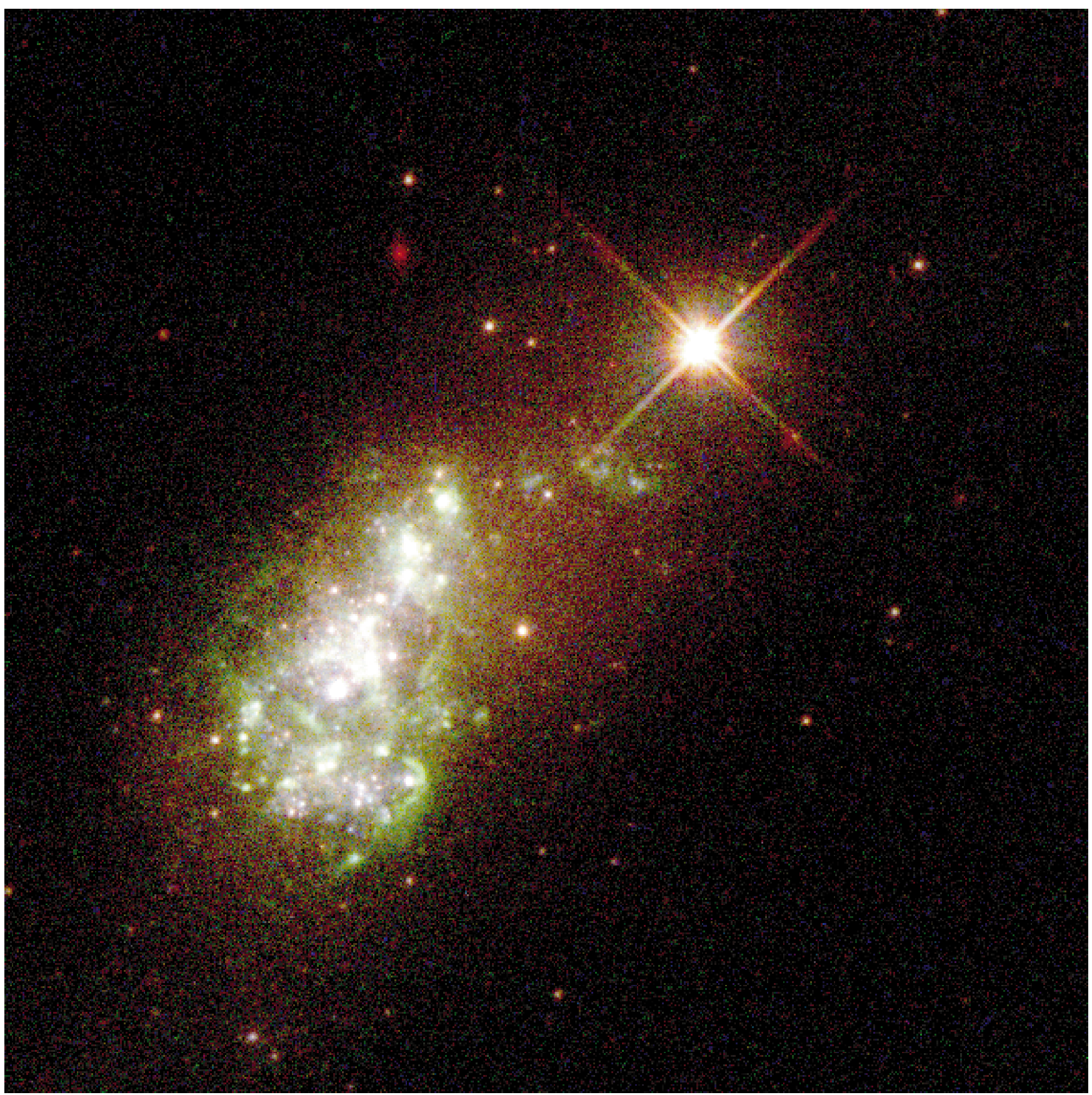

Fig. 1. True colour image composite of the central $30^{\prime \prime} \times 30^{\prime \prime}$ (corresponding to $5.45 \times 5.45 \mathrm{kpc}$ at the adopted distance $37.5 \mathrm{Mpc}$ ) region of the Planetary Camera. F814W $(i)$ is coded in red, F555W $(v)$ in green and F439W $(b)$ in blue. North is up-left (65 degrees measured from vertical towards left) and east is down-left. The bright source with diffraction spikes $\sim 5^{\prime \prime}$ from the centre towards the upper right is a Galactic foreground star.

others resemble compact star clusters, which is confirmed by our new deep STIS data (Östlin et al. in prep.). The STIS images $($ FOV $9.3 \times 9.3 \mathrm{kpc}$ ) also show a small number of bright clusters lying outside the upper and right boundaries of the PC FOV. Bergvall \& Östlin trace the luminosity profile of ESO 338-04 to radii greater than $10 \mathrm{kpc}$, and in ÖBR98 it was shown that the radial cluster distribution follows that of the visual light. From this we estimate that the limited area of the PC results in a cluster incompleteness, at the $\sim 11 \%$ level, in addition to the photometric incompleteness.
In Table 3 we present the integrated starburst luminosity in different passbands and the fraction of light attributable to compact star clusters.

\section{Spectral evolutionary synthesis models}

Due to the uncertainties still prevailing the field of spectral evolutionary synthesis, all results should ideally be cross-checked with independent models. To ensure that the age and mass distributions derived for our star cluster sample do not critically 


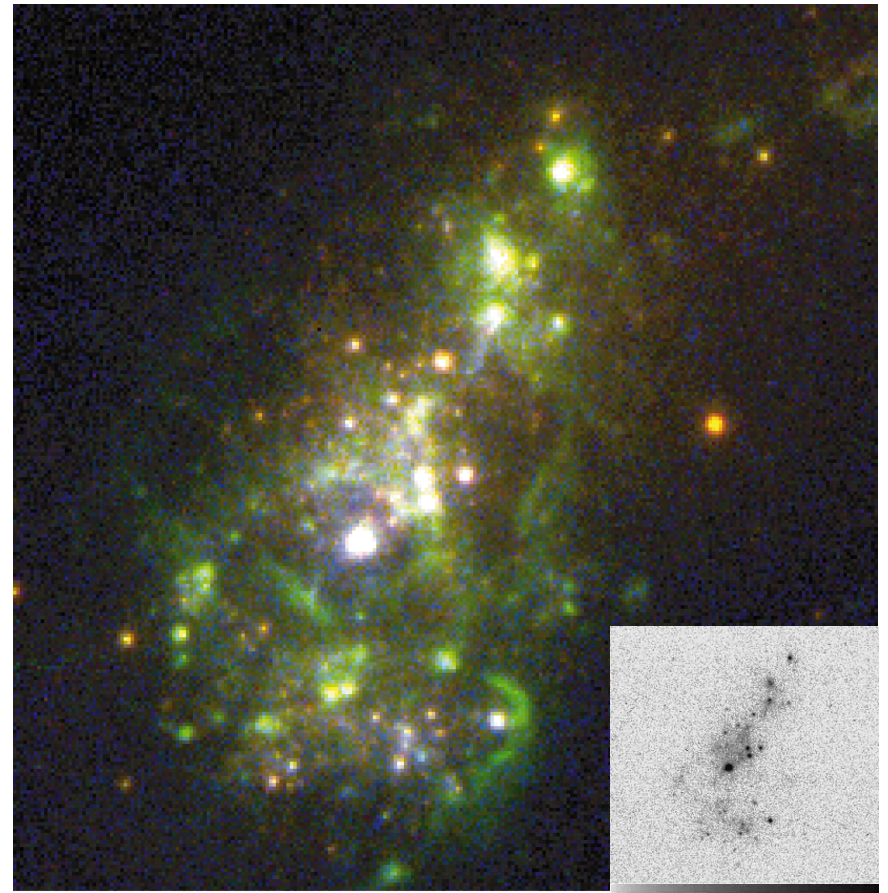

Fig. 2. True colour RGB composite of the central 11.6" $\times 11.6^{\prime \prime}(2 \times$ $2 \mathrm{kpc}$ ) region with cuts chosen to show details of the central bright regions: $\mathrm{F} 814 \mathrm{~W}(i$, red), $\mathrm{F} 555 \mathrm{~W}(v$, green) and $\mathrm{F} 218 \mathrm{~W}(x$, blue). The greyscale inset shows the F218W image in of the same field.

depend on the predictions from any single code, the analysis has been carried out with two models in parallel: PÉGASE.2 (Fioc \& Rocca-Volmerange 1999) and the model by Zackrisson et al. (2001, hereafter Z2001). These particular models were chosen since they contain a nebular component and because of the ease with which non standard filters may be included.

PÉGASE. 2 is based on the method of isochrone synthesis, employs stellar atmospheres by Lejeune (1998) and Clegg \& Middlemass (1987), together with stellar evolutionary tracks mainly from the Padova group. Using a simple prescription for the nebular component, it predicts both continuum and line emission of the ionised gas.

Z2001 is based on the method of isomass synthesis, employs the same set of stellar atmospheres as PÉGASE.2 but stellar evolutionary tracks mainly from the Geneva group. Premain sequence evolution and a stochastic treatment of horizontal branch morphologies at low metallicities are also included. Gas continuum and emission lines are predicted using the photoionisation code Cloudy version 90.05 (Ferland 1996). For each time step, the spectral energy distribution of the stellar population is fed into Cloudy, making the computation time consuming, but also giving a more realistic nebular emission component.

The use of pre-main sequence evolution and and a more sophisticated nebular component in Z2001 makes this model more suited for the current analysis, where many clusters are believed to be young. As will be demonstrated (see Sects. 4.1 and 5.1) the predictions by Z2001 are also in better agreement with the observations. A large grid of evolutionary sequences was therefore generated using Z2001, and a smaller set,

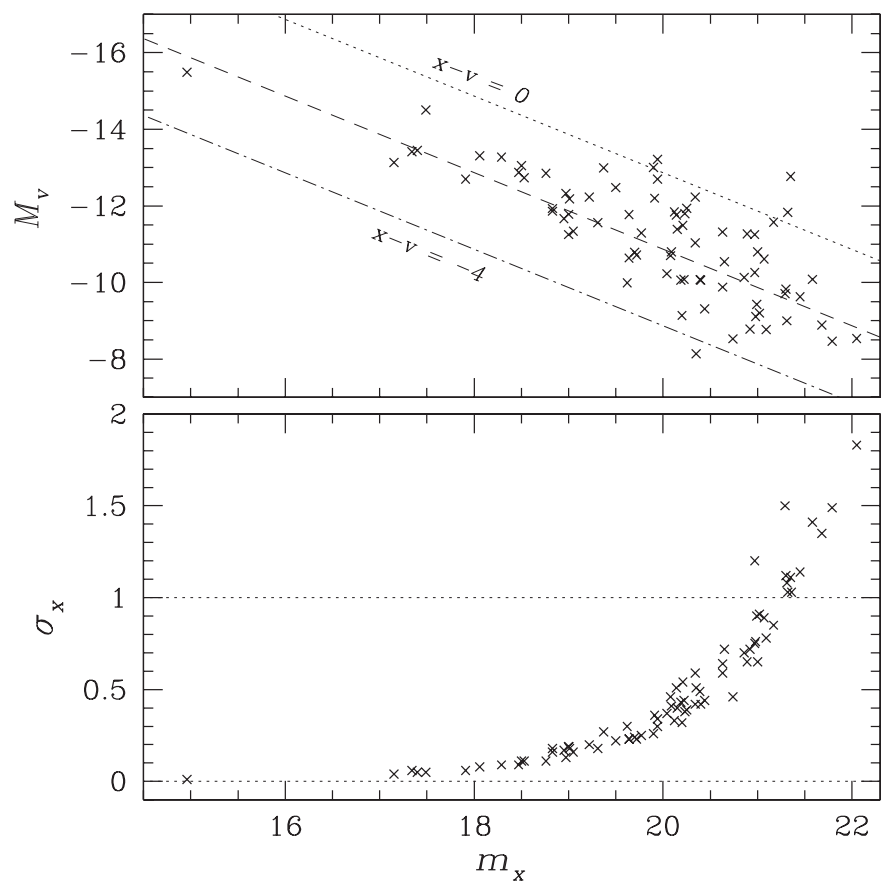

Fig. 3. Photometry for clusters detected in the F218W $(x)$ band. The bottom panel shows the magnitude-uncertainty diagram. Top panel shows the apparent $x$ magnitude vs. the absolute $v$ band magnitude. Lines of constant colour are shown as well: $x-v=0$ (dotted), -2 (dashed), and -4 (dash-dot).

intended to use as a consistency check, with PÉGASE.2. The details of these sequences are given in Tables 1 and 2. In total, 133 parameter configurations were processed with Z2001, 10 with PÉGASE.2. The two models used represent an improvement over the one used in ÖBR98 in terms of the nebular component, time resolution at young ages, updated stellar evolutionary tracks and stellar atmospheres at a wider range of metallicities. In addition, the Z2001 grid represents an increase in the size of the parameter space by more than a factor of 30 compared to ÖBR98.

Magnitudes in the HST Vegamag system were generated from model spectra using the HST/WFPC2 filter+CCD response curves, and a theoretical Vega model. Since the full transmission profiles were used, red leaks are included in the calculation and do not result in any degenercies. The stellar initial mass function (IMF) was parametrised as a power-law: $\mathrm{d} N / \mathrm{d} M \propto M^{-\alpha}$, with spectral index $\alpha$. For a Salpeter (1955) IMF, $\alpha=2.35$. The Scalo (1998, hereafter Scalo98) IMF used in some of the PÉGASE.2 evolutionary sequences was parametrised by a piecewise continuous 3 segment power-law.

\section{Modelling the ages of star clusters}

The object magnitudes (corrected for aperture effects, Galactic extinction, and UV-contamination) were converted into fluxes and assigned weights inversely proportional to the square of the uncertainties. Since the uncertainties are based on the photon statistics, and there are other error sources present as well, e.g. zero-point and flat field errors, a maximum weight corresponding to an uncertainty of $0.03 \mathrm{mag}$ was used. 


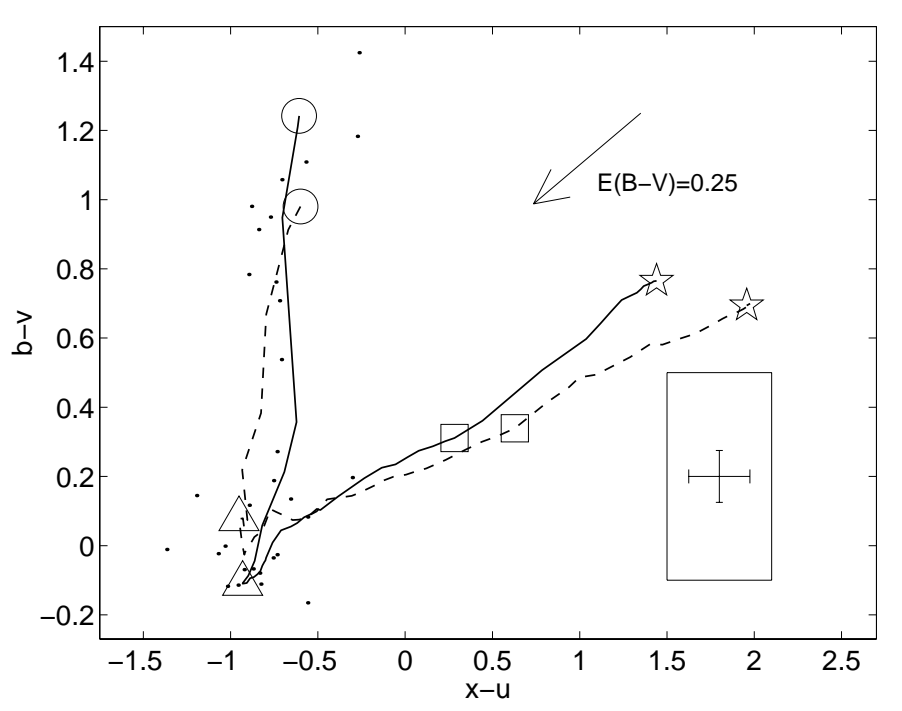

Fig. 4. Colour-colour diagram including the F218W data (denoted "x"). The median colour uncertainty for objects included in the plot is shown as a cross in the lower right, whereas the largest uncertainty associated with any object is indicated by the surrounding rectangle. The arrow indicates how much and in what direction the objects would be repositioned after correction for a hypothetical extinction of $E(B-V)=0.25$. The predictions for two of the used spectral evolutionary synthesis models (Salpeter IMF, $M=0.08$ to $120 M_{\odot}$, instantaneous burst, $\left.Z_{\text {gas }}=0.002, Z_{\text {stars }}=0.001\right)$ are shown as well: Zackrisson et al (2001, solid line), and PÉGASE.2 (dashed line). Markers indicate ages of $0.5 \mathrm{Myr}$ (circles), $10 \mathrm{Myr}$ (triangles), 1 Gyr (squares) to $15 \mathrm{Gyr}$ (pentagrams).

The contribution from emission lines is significantly larger in the $v$ filter $(\mathrm{F} 555 \mathrm{~W})$. Since the nebular component is sensitive to the assumed ISM parameters, and because of the difficulty in isolating all the diffuse nebular emission associated with a particular star cluster, we increased the formal uncertainties with a factor of 2 in this filter. The predictions from the spectral synthesis models, having 51 time steps for the Z2001 model, is interpolated linearly onto a finer grid with an age resolution of 1 Myr up to 3 Gyr and 100 Myr from 3 to 15 Gyr. Finally, we find the best fitting age for each cluster by minimising the weighted rms deviation. When allowing $E(B-V)$ to vary, we used $E(B-V)=0.0,0.05,0.10,0.15,0.20$ and 0.25 and determined the best fitting value for each cluster.

Masses were determined by scaling the extinction corrected $v$ flux to the $M / L_{v}$ predicted by the model. These take into account the total mass converted into stars and thus accounts also for stellar remnants and gas returned in the process of stellar evolution. The "true" stellar mass (excluding returned gas but including remnants) will therefore be overestimated at high ages, but since we are interested in the star formation history, we prefer using the total mass initially present in each cluster. At $15 \mathrm{Gyr}$ the overestimate in $M / L_{v}$ will amount to a factor of $\sim 1.5$ for $0.08-120 M_{\odot}$ Salpeter IMFs (for any $Z_{\text {stars }}$ ), $\sim 3$ for $\alpha=1.35$, and $\sim 1.1$ for $\alpha=2.85$.

In Fig. 5 we show the $v-i$ vs. $b-v$ diagram for clusters with errors smaller than 0.15 mag in both colours together with a predictions from the Z2001 and PÉGASE. 2 codes for a standard set of parameters. Only Z2001 is successful in reproducing the youngest clusters, emphasizing the need for a careful
Table 1. The grid of Z2001 (Zackrisson et al. 2001) evolutionary sequences. The grid consists of all possible combinations of the parameter values listed below. To furthermore ensure the robustness of the final results, a smaller number of evolutionary sequences was also calculated with variations like: constant star formation during $5 \mathrm{Myr}$ and $100 \mathrm{Myr}, M_{\text {up }}=40,60,80 M_{\odot}$, $10^{6} M_{\odot}$ available for star formation, $n(\mathrm{H})=100 \mathrm{~cm}^{-3}$, filling factor $1.0, Z_{\text {stars }}=0.004,0.008,0.020,0.040$ and $Z_{\text {gas }}=$ $0.001,0.004,0.008,0.020,0.040$. Neither the variations in hydrogen density, filling factor nor stellar mass were seen to have any serious impact on the final ages and masses derived.

\begin{tabular}{ll}
\hline \hline IMF, $\alpha$ & $1.35,2.35,2.85$ \\
$M_{\text {low }}$ & $0.08,2 M_{\odot}$ \\
$M_{\text {up }}$ & $20,120 M_{\odot}$ \\
$S F H$ & $\mathrm{c} 1 \mathrm{e} 7, \mathrm{e} 3 \mathrm{e} 6$, Inst \\
$Z_{\text {stars }}$ & 0.001 \\
$Z_{\text {gas }}$ & 0.002 \\
$M_{\text {stars }}$ & $10^{5} M_{\odot}$ \\
$n(\mathrm{H})$ & $10 \mathrm{~cm}^{-3}$ \\
Filling factor & 0.1 \\
Covering factor & $0.5,1.0$ \\
Nebular emission & Yes, No \\
\hline
\end{tabular}

IMF: $\mathrm{d} N / \mathrm{d} M \propto M^{-\alpha}$.

SFH = Star Formation History: c1e7 = constant SFR during $10 \mathrm{Myr}$, e3e6 = exponentially declining SFR with e-folding decay rate of $3 \mathrm{Myr}$, Inst = Instantaneous burst.

Table 2. List of used PÉGASE.2 evolutionary sequences, all assuming $Z_{\text {stars }}=Z_{\text {gas }}=0.002$. All evolutinary sequences were computed both with and without inclusion of nebular emission.

\begin{tabular}{llll}
\hline \hline IMF & $M_{\text {low }}\left(M_{\odot}\right)$ & $M_{\text {up }}\left(M_{\odot}\right)$ & SFH \\
\hline Salpeter & 0.1 & 120 & Inst \\
Salpeter & 0.1 & 20 & Inst \\
Salpeter & 0.1 & 120 & e3e6 \\
Salpeter & 1 & 120 & Inst \\
Scalo 1998 & 0.1 & 120 & Inst \\
\hline
\end{tabular}

treatment of the nebular contribution. For ages larger than a few times $10 \mathrm{Myr}$, both models approximately perform equally well. However, around a few 10 Myrs the PÉGASE. 2 model presents loops in the colour-colour diagram (Fig. 5) which the Z2001 model does not. This different behaviour is due to the use of different evolutionary tracks (see Charlot et al. 1996 for a discussion).

\subsection{Internal reddening}

The effects of age and reddening are in general difficult to disentangle, although the reddening and aging vectors are not absolutely parallel. In particular, the reddening vector for young objects is close to orthogonal to the age-metallicity sequence (Fig. 5) and, as has also been shown by other investigators (e.g. Mas-Hesse \& Kunth 1999), the addition of $2000 \AA$ data helps in deriving the extinction for very young objects, since the UVspectral slope is nearly constant at low ages (see Fig. 4). 
Table 3. Integrated starburst luminosities. All values have been corrected for foreground reddening by our Galaxy. The first column gives the name of the filter, and the second column the effective wavelength of the filter+CCD combination (Biretta et al. 2002). The third column gives the integrated starburst magnitude (in the VEGAMAG system) within a radius of $5.9^{\prime \prime}(1.075 \mathrm{kpc})$ centred on the brightest cluster. The fourth column gives the same quantity in $f_{v}$ units. The fifth column gives the magnitude of the starburst region after all detected compact clusters have been subtracted. The last column gives fractional contribution from compact star clusters to the integrated starburst luminosity.

\begin{tabular}{llllll}
\hline \hline Filter & $\langle\lambda\rangle$ & \multicolumn{2}{l}{ Starburst } & Diffuse & $L_{\mathrm{cl}} / L_{\mathrm{SB}}$ \\
\cline { 3 - 4 } & & mag & $f_{v}$ & $\begin{array}{l}\text { mag } \\
\text { vega }\end{array}$ \\
& $\AA$ & vega & $\mathrm{W} / \mathrm{m}^{2} / \mathrm{Hz}$ & \\
\hline$x(\mathrm{~F} 218 \mathrm{~W})^{a}$ & 2210 & 12.61 & $6.6 \times 10^{-29}$ & 12.97 & 0.29 \\
$u(\mathrm{~F} 336 \mathrm{~W})^{a}$ & 3348 & 13.35 & $5.7 \times 10^{-29}$ & 13.66 & 0.25 \\
$b(\mathrm{~F} 439 \mathrm{~W})$ & 4316 & 14.58 & $6.3 \times 10^{-29}$ & 14.83 & 0.21 \\
$v(\mathrm{~F} 555 \mathrm{~W})$ & 5465 & 14.08 & $8.7 \times 10^{-29}$ & 14.31 & 0.19 \\
$i(\mathrm{~F} 814 \mathrm{~W})$ & 8040 & 14.07 & $5.9 \times 10^{-29}$ & 14.37 & 0.15 \\
\hline
\end{tabular}

Note: a - corrected for UV-contamination 0.12 mag in F228W and 0.01 mag in F336W.

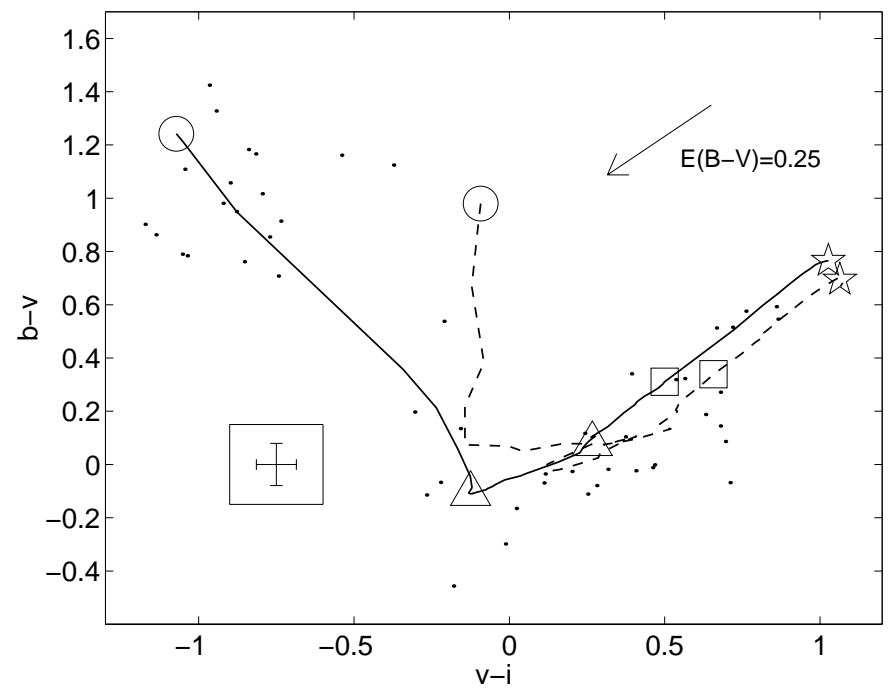

Fig. 5. Colour-colour diagram ( $v-i$ vs. $b-v)$, see caption of Fig. 4 for more details. The full drawn line show the prediction form the Z2001 model with Salpeter IMF, $M=0.08$ to $120 M_{\odot}$, instantaneous burst, covering factor $=1.0, Z_{\mathrm{gas}}=0.002, Z_{\mathrm{stars}}=0.001$. The dashed line shows the prediction from the publicaly available PÉGASE. 2 model with similar model parameters (instantaneous burst with 0.08-120 $M_{\odot}$ Salpeter IMF, $Z_{\text {gas }}=0.002, Z_{\text {stars }}=0.002$ ).

In ESO 338-IG04 we have several reasons to suspect that the internal reddening is very small. Ground based studies of the Balmer emission line decrement (Bergvall 1986; Iye et al. 1987; Calzetti et al. 1994; Raimann et al. 2000), as well as studies of the UV continuum (Calzetti et al. 1994; Meurer et al. 1995; Buat et al. 2002) of the central star forming region have given values of $E(B-V)$ in the range from 0 to 0.05 . Further support from this conclusion comes from a comparison of the far IR to UV luminosity (Meurer et al. 1999; Buat et al. 2002) and the colours of the bluest star clusters themselves (this paper). Hence, we know that the average extinction is very small, making ESO338-IG04 an ideal target to study the formation and evolution of star clusters. However, star formation is normally associated with dusty molecular clouds, and it is likely that the reddening varies from cluster to cluster. Especially we may suspect the existence of embedded very young sources, that, however, will have quite low probability of being picked out from this optically selected sample. Ground based long-slit spectra, obtained with the ESO NTT shows a $\mathrm{H} \alpha / \mathrm{H} \beta$ value that varies along the slit, corresponding to a varying $E(B-V)$ in the range 0.0 to 0.20 , although the luminosity weighted average is close to zero. To adress the internal extinction we have adoped two approaches: i) Allowing $E(B-V)$ to be a free parameter in the fit, but constraining it to the range $E(B-V) \in[0-0.25]$. ii) Adopting a single extinction for all clusters in the range $E(B-V) \in[0-0.25]$.

In general we use a Galactic extinction law (Seaton 1979), which has an intermediate behaviour in the UV compared to the LMC and Calzetti laws commonly used for starbursts (Calzetti 1994). In the optical, the difference is small. Tests perfomed with the Calzetti law give close to identical results regarding age and best fitting $E(B-V)$. Hence, the results presented are not sensitive to the choice of extinction law within the range of low extinctions considered. However, for modelling the UV emission of clusters in more reddened galaxies, the extinction law will be an additional source of uncertainty.

\subsection{Comments on the parameter choices}

In most model runs, we have kept the parameter choice fixed, but in some cases, we have allowed some parameters to be free, as e.g. the covering factor or the IMF slope. There is accumulating evidence for an IMF slope very close to the Salpeter value for stars more massive than $1 M_{\odot}$ (Massey \& Hunter 1998; Kroupa 2002; Kennicutt 1998; Leitherer 1998). At lower masses, the mass function seems to turn-over with the result that the IMF can be approximated by a multi-segment powerlaw (e.g. Miller-Scalo 1982; Scalo 1998). Since the main sequence life time of a $1 M_{\odot}$ star is on the order of $10 \mathrm{Gyr}$, the shape of the lower mass IMF will have very little influence on colours for any age considered in this paper. The main effect lies in a different mass normalisation leading to lower effective $M / L$ values for the Scalo98 (Scalo 1998) IMF for ages greater than $300 \mathrm{Myr}$, compared to a Salpeter IMF.

The choice of IMF slope $(\alpha)$, upper mass limit and covering factor has important effects on the colours at low ages. At high ages, the most important effect is on the mass to light $(M / L)$ values, introducing uncertainties in the masses estimated from photometry.

\subsubsection{Covering factor}

In our Z2001 model runs we have used two different gas covering factors in the calculation of the gas spectrum: 1.0 and 0.5 , describing what fraction of ionising photons along the line of sight that will be absorbed. This influences the strength of emission lines relative to the continuum, and hence the 
predicted flux, notably in the $v$ filter. A covering factor of 0 , corresponding to no nebular emission contribution at all, was also considered, but could not fit the data for young/blue objects. The choise of 0.5 is somewhat arbitrary, but was included to have an intermediate value to get an estimate of the importance of this parameter.

Figure 1 shows that a fraction of the ionised gas emission is found outside the bright clusters, but this is expected since even in the UV, young clusters don't produce more than $30-40 \%$ of the starburst luminosity. The ionising continuum from a young cluster will produce significant nebular emission, that may have a different spatial distribution compared to the clusters. Narrow-band [O III] images obtained with HST/STIS (Östlin et al. in prep.) show that the peaks in the [O III] surface brightness indeed coincide with blue UV-luminous sources. However, the [O III] is more diffuse than the stellar continuum, and our apertures could miss a fraction of the gas emission. Due to the ionisation structure, a larger fraction of the [O III] emitting zone will be included as compared to the [O II] and HII zones. Since our apparently youngest clusters still have as blue $(v-i)$ and red $(b-v)$ colours (where the $v$ band excess mainly comes from [O III] line emission) as the earliest model steps, the net contribution from gas outside the aperture must be small.

Maiz-Appellaniz (2001) studied the spatial structure of gas emission in a sample of young star clusters, and found that $\sim 2$ Myr old clusters have small ( $\sim 10 \mathrm{pc})$ shells around them, whereas $\sim 4$ Myr clusters have larger shells, and for older clusters only diffuse $\mathrm{H} \alpha$ emission is detectable. This indicates that with photometric aperture diameters of $\sim 50 \mathrm{pc}$, we would not miss any emission during the first few Myr, and the covering factor should be close to unity. At later times (5-10 Myr), we may miss a significant fraction of the emission, which can be approximated by a smaller covering factor. At still larger ages the clusters will be too cool to ionise [O III] and the ionisation structure will be of little concern. Moreover, the relative gas contribution will be almost negligible in all passbands used here.

\subsubsection{Metallicity}

The nebular oxygen abundance in ESO 338-IG04, as determined using the temperature sensitive $[\mathrm{OIII}]_{\lambda 4363}$ line, has been shown to be on the order of 10-14\% solar (Bergvall 1984; Masegosa et al. 1994). Hence, in general, we adopt $Z_{\text {gas }}=$ $0.002,\left(Z_{\odot}=0.0188\right)$. Other values (see Table 1$)$ are also included to test how the assumed nebular abundances affect the results. It is comforting that the best fit is provided by $Z_{\text {gas }}=0.002$.

For the stars, we have very limited information on the metallicity. In general $\mathrm{Fe}$, which is the dominant source of stellar opacity, is less abundant (compared to solar) than $\mathrm{O}$ in metal-poor galaxies (Kunth \& Östlin 2000). Also, in a closed box model, the stars should on average have lower metallicity than the gas. Hence, the choice $Z_{\text {stars }}=0.001$ seems reasonable. However, there are indications from near-IR surface photometry that the underlying old host galaxy may be more metal rich than the nebular gas (Bergvall \& Östlin 2002). This intriguing result can be interpreted as metal-poor gas being accreted onto a more metal-rich host galaxy in a merger. Whereas young objects should have a metallicity close to that of the gas, it cannot be excluded that older clusters are significantly more metal-rich. However, metal-rich elliptical galaxies have in general metal-poor (typically $[\mathrm{Fe} / \mathrm{H}]<-1$, Brodie \& Huchra 1991) old globular cluster systems. The commonly found metal-rich sub-populations are in general younger and still less metal-rich than the host galaxy (Ashman \& Zepf 1998).

\subsubsection{Star formation time-scale}

The assumed time-scale for star formation $\tau_{\mathrm{SF}}$, i.e. how long star formation is active in a particular event, impacts the model predictions for ages comparable to $\tau_{\mathrm{SF}}$. As long as star formation is active, young stars will make colours blue and modelled ages tend to increase with the assumed $\tau_{\mathrm{SF}}$. This is a major source of uncertainty when modelling the integrated light from galaxies. In these compact ( $r \sim$ a few pc) star clusters we know that $\tau_{\mathrm{SF}}$ must be short, which is a main reason why ages of such are less degenerate than those for unresolved populations. Of course, the common assumption of a truly instantaneous formation of a massive star cluster is unrealistic. Another question is whether low- and high-mass stars form simultaneously, or with some time lag inbetween. When massive stars appear, they ionise their surroundings and inhibit further star formation. Hence, low mass stars must form prior to or simultaneously with the massive stars. McKee \& Tan (2002) argues that the formation time scale for individual stars is short, of the order of $10^{5} \mathrm{yrs}$, permitting the coeval formation of low and high mass stars in a cluster. Massey \& Hunter (1998) found that the formation of R136, the central cluster in 30 Doradus, might have been extended over $\sim 4$ Myrs, with low mass stars forming prior to massive ones. Moreover the IMF slope is $\alpha=2.3-2.4$ over the studied range $2.8-120 M_{\odot}$, i.e. identical to the Salpeter (1955) value. Similarly, the central star cluster in the Galactic giant HII region NGC 3603 shows an age spread of a few Myr (Pandey et al. 2000).

Our three main SFHs considered are instantaneous burst, a constant SFR for 10 Myr after which the SFR drops to zero, and an exponentially declining SFR with an e-folding time of 3 Myr. A smaller number of other parameter options were tested as well (Table 1). A potential drawback with the exponential model is that the SFR only goes asymptotically to zero, and hence remains significant for quite a long time. Although little of what is derived in this paper will be substantially changed whether one adopts either of these three timescales, we will show below that comparison with the global $\mathrm{H} \alpha$ output tend to favour the shorter one. An instantaneous burst also gives on average lower rms residuals.

In all our evolutionary sequences, low- and high-mass stars form simultaneously. If in reality there is a time lag of a few Myr in the formation of the most massive stars, this will influence our results for ages on the order the time lag. For a time lag of 3 Myr our model would behave as follows: looking at Fig. 8 for reference, the time-lag tracks would start near 
$v-i=-0.1, b-v=0$ and climb along the simultaneous tracks up to $v-i=-1.2, b-v=1.2$, a point it would reach when the most massive stars appear after 3 Myr. Then the spectrum would essentially follow the same track as for the simultaneous case model. Hence the only effect would be that we would confuse ages during the first $3 \mathrm{Myr}$. We will discuss the possible implications of time-lag on our results when relevant.

\subsection{Monte-Carlo simulations}

In order to assess the internal accuracy of the fitted ages and extinctions, we constructed catalogues of artificial clusters with the same age and luminosity distribution as indicated by the model fits and with colours that perfectly fit the models. To these ideal clusters we added observational errors in accordance with the observed error distribution. Then this fake catalogue was fitted to the models. The experiment was repeated 100 times to beat down the statistical noise and from the dispersion of the fitted ages we could for each object assess the uncertainty in the derived age, for the assumed parameter configuration in question. The top two panels in Fig. 6 show the input (top) and output (second row) age and $E(B-V)$ distributions for a sample Monte-Carlo experiment. The tendency to slightly overestimate the output extinction is statistically significant, but is only a few hundredths of a magnitude on average.

We also created fake catalogues with fixed input $E(B-V)$ values. Specifically, we wanted to test whether the $E(B-V)$ range found from the free fit could be an artifact produced from an (in reality) single reddening value smeared out by observational errors. The bottom panel in Fig. 6 compares the reddening found from the free fit method to simulations with fixed input $E(B-V)$. Obviously, no single reddening value can be found which can reproduce the distribution found when letting $E(B-V)$ be a free parameter, although $E(B-V)=0$ comes quite close. Hence, we conclude that there is a real spread in $E(B-V)$, but letting it be a free parameter on average may lead to a slight overestimate of the true $E(B-V)$.

Since the colour and luminosity of a cluster changes with time, the uncertainty in the derived ages is time dependent. We have used the Monte-Carlo simulations to estimate the internal uncertainties on the derived ages resulting from observational errors. The net effect is small uncertainties $\left(\sigma_{\text {Age }} \approx 0.1 \mathrm{dex}\right)$ for ages less than $100 \mathrm{Myr}$, considerable uncertainties in the range 100 Myr to $1 \mathrm{Gyr}(\approx 0.4 \mathrm{dex})$, and again quite small uncertainties for ages greater than $1 \mathrm{Gyr}(\approx 0.2 \mathrm{dex})$. These numbers are similar for any evolutionary sequence with $Z_{\text {stars }} \leq 0.004$. For higher metallicities the age of many objects decreases, and the region of higher uncertainties at intermediate ages is shifted to smaller ages. Our conlusion is that the overall age pattern is quite insensitive to observational errors.

\section{Results}

We find an overall temporal age pattern that is quite robust to changes in all model parameters except $Z_{\text {stars }}$ and the lower mass limit. The latter matter only for ages comparable to the lifetime of stars with mass equal to $M_{\text {low }}$, and $M_{\text {low }}=2 M_{\odot}$ is unrealistic for ages $>1$ Gyr. Despite some claims of IMFs
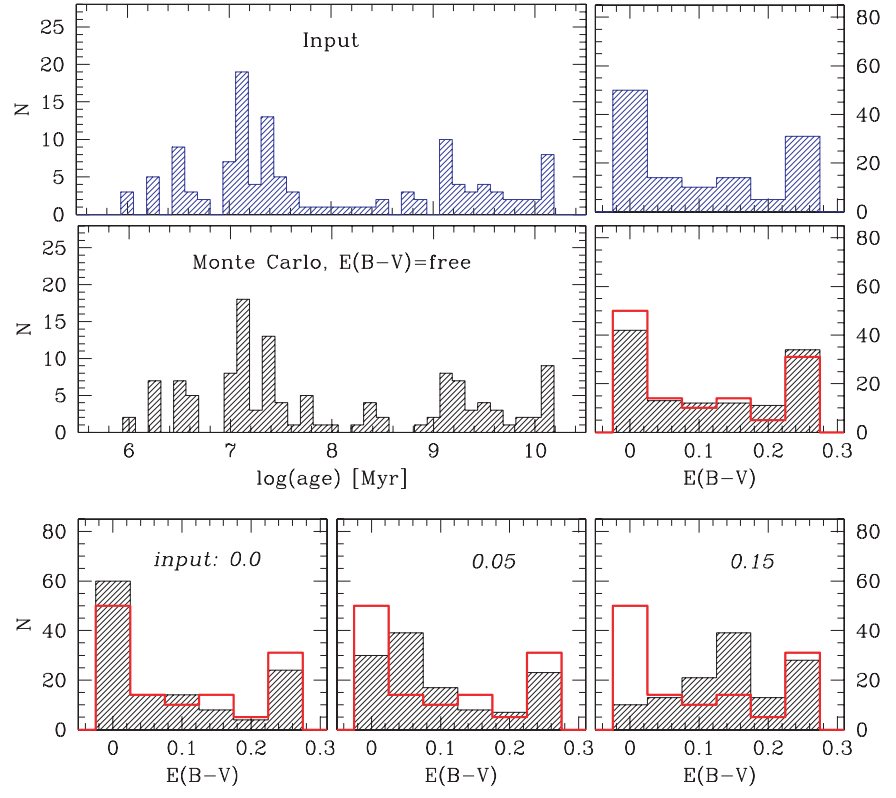

Fig. 6. Some results from the Monte-Carlo simulations. Top row: results from a typical evolutionary sequence (Salpeter IMF, $M=0.08$ to $120 M_{\odot}, 10 \mathrm{Myr}$ burst with constant SFR, covering factor 1.0, $\left.Z_{\text {gas }}=0.002, Z_{\text {stars }}=0.001\right)$. On the left we show the age distribution, and on the right the $E(B-V)$ distribution. This age and $E(B-V)$ distribution was used to create a fake catalogue where the objects match the evolutionary sequence perfectly in all filters. To this fake catalogue, random errors were added according to the observed uncertainties, and then used as input to the fitting program. Second row: sample output from this experiment when $E(B-V)$ was a free parameter. In the $E(B-V)$ histogram, the "input" $E(B-V)$ distribution of the top panel is shown as a thick unshaded histogram. Third row: output $E(B-V)$ distributions from sample Monte-Carlo simulations where the input fake catalogue was created with fixed values of $E(B-V)$. The input $E(B-V)$ value is given as a number in each sub-panel, and the $E(B-V)$ distribution of the top panel is shown as a thick unshaded histogram for reference.

devoid of low mass stars in starburst regions, there is no direct evidence for such, and we mainly consider such evolutionary sequences for comparison.

In Fig. 7 we show age and mass histograms for a selection of evolutionary sequences with $E(B-V)$ as a free parameter and fixed $E(B-V)=0.05$. In accordance with ÖBR98, there is evidence both for young (less than $100 \mathrm{Myr}$ ) intermediate (a few Gyr) and old (more than 10 Gyr) clusters if a low metallicity is adopted. If a high metallicity is adopted, the net effect is to compress the age span, but retaining the main features. A metal-rich stellar component provides a poor fit to the bluest objects which must therefore have a metallicity close to that derived for the nebular gas. Adopting a nebular metallicity of $Z_{\text {gas }}=0.002$, provides for a significantly better fit for young clusters, which give us confidence in our way of handling the nebular emission in Z2001.

The shape of the mass histograms are model dependent (due to the different mass normalisations inherent to each IMF), but for most parameter choices we find that the maximum mass is higher for clusters older than $1 \mathrm{Gyr}$, and more mass seems to be found in relatively old than young clusters. All 

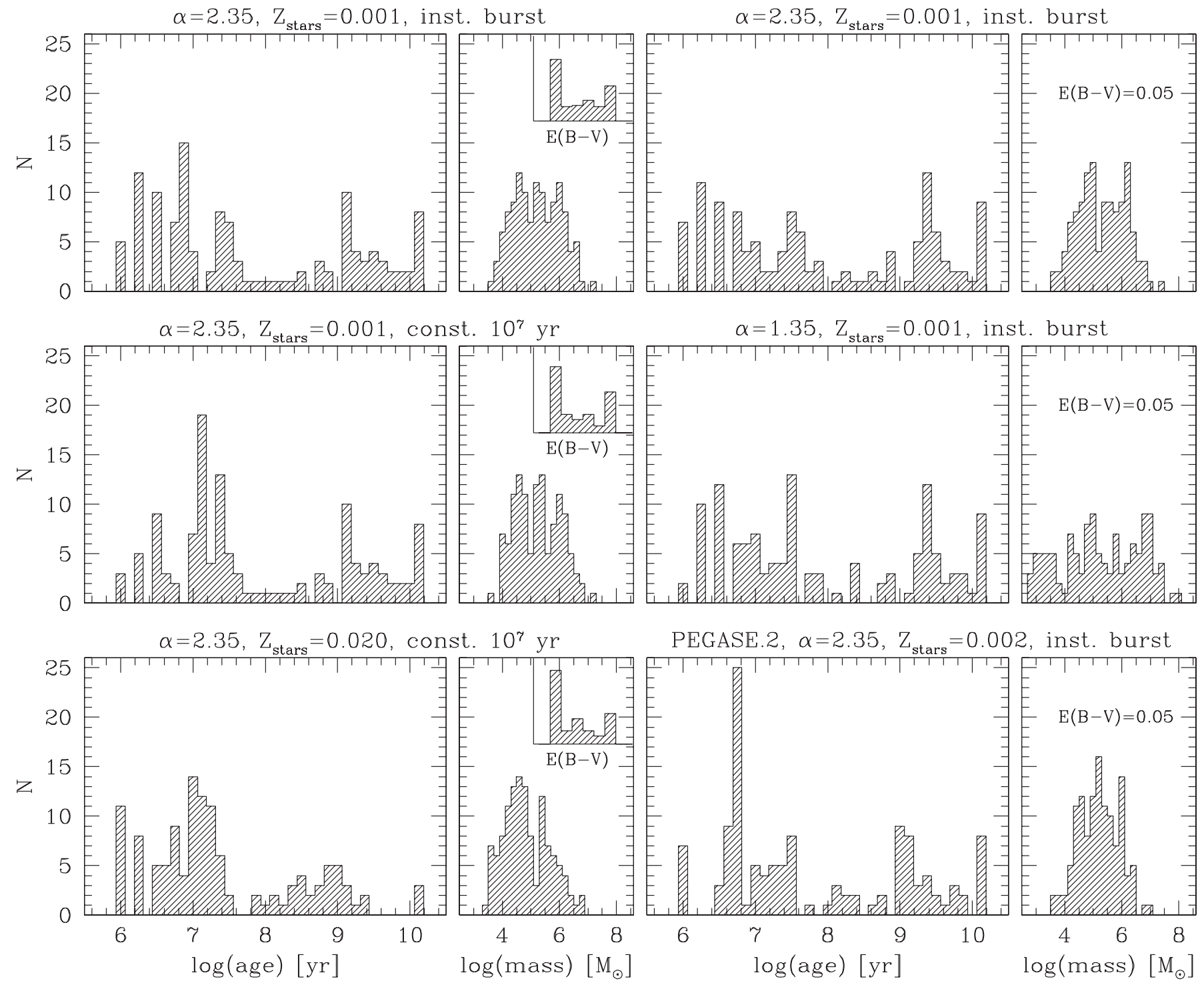

PEGASE.2, $\alpha=2.35, \mathrm{Z}_{\text {stars }}=0.002$, inst. burst

Fig. 7. Comparison of output age and mass distributions for different model assumptions. In the left panels, the extinction was allowed to vary freely in the range $E(B-V) \in[0-0.25]$, and the resulting exinction distribution is indicated by the inset in the mass histograms. In the right panels a fixed extinction of $E(B-V)=0.05$ was used. All evolutionary sequences, except for the lower right one, was calculated with Z2001 code, and have the following parameteers in common: Mass range $M=0.08$ to $120 M_{\odot}, Z_{\text {gas }}=0.002$, and covering factor 1.0 . Other parameters: top left: Salpeter IMF $(\alpha=2.35), Z_{\text {stars }}=0.001$ and instantaneous burst. middle left: $\alpha=2.35, Z_{\text {stars }}=0.001$ and constant SFR for 10 Myr. lower left row: $\alpha=2.35, Z_{\text {stars }}=0.020$ and constant SFR for 10 Myr. top right: Salpeter IMF $(\alpha=2.35), Z_{\text {stars }}=0.001$ and instantaneous burst. middle right: $\alpha=1.35, Z_{\text {stars }}=0.001$ and instantaneous burst. lower right: PEGASE. 2 model $\alpha=2.35, Z_{\text {stars }}=0.002$ and instantaneous burst.

evolutionary sequences give a mass function (MF) with many clusters with $M>10^{6} M_{\odot}$, showing that this galaxy is rich in massive globular clusters.

Comparing the free fit $E(B-V)$ with the $E(B-V)=0.05$ results (top two panels in Fig. 7), one sees that peaks in the age distribution tend to be displaced to lower ages when $E(B-V)$ is allowed to vary freely. This effect is notable for the peak at an age of a few Gyr. The peak is there for all metal-poor evolutionary sequences but occur at 0.2 dex lower age for the free fit $E(B-V)$ cases. At these ages, few sources have accurate photometry in more than 2 bands ( $v$ and $i$ ) and the fixed $E(B-V)$ results are probably more robust and less prone to systematic errors. However, the overall results in terms of age and mass distributions are insensitive to the adopted extinction treatment, but this is so only because we have a priori information that the extinction must be low.

When adopting a fixed $E(B-V)=0.05$ (as in ÖBR98) we find a good overall agreement with the result in ÖBR98, except for ages below $10 \mathrm{Myr}$ where the evolutionary sequences used in ÖBR98 had no time resolution. We do not find the peaks at 100 and $600 \mathrm{Myr}$ reported by ÖBR98, although we do find objects with these ages.

The age pattern is sensitive to the adopted star formation time-scale $\tau_{\mathrm{SF}}$ at ages comparable to $\tau_{\mathrm{SF}}$ (compare top-left and mid-left panels in Fig. 7). The IMF influences the ages, but the effect is small at high ages; the effect on masses is more severe 
(upper and middle right panels in Fig. 7). Both the $\alpha=1.35$ and 2.85 evolutionary sequences have problems at high ages since the predicted mass to light ratios (for $15 \mathrm{Gyr}: M / L_{V}=47$ and 8.7, respectively, as measured by the total consumed mass, whereas $\alpha=2.35$ give $M / L_{V}=6^{2}$ or $M / L_{V}=4.2$ if counting only stars and remnants) is much higher than those observed in old Galactic globular clusters $\left(M / L_{V} \sim 2\right.$, Ashman \& Zepf 1998). Gas parameters are only significant for ages smaller than or comparable to a few times $\tau_{\mathrm{SF}}$. The found $E(B-V)$ distribution is relatively insensitive to the adopted parameters. Within each parameter configuration we have analysed the correlation between the fitted age, mass, residual and $E(B-V)$. Mass and age are corellated (as expected since $M / L$ is a strong function of age), but not the other quantities.

We have performed correlation analysis on the output from the different parameter configurations. All evolutionary sequencecs with a low stellar metallicity and $M_{\text {low }}=0.08 M_{\odot}$ show a high degree of mutual correlation, corroborating the results in Fig. 7. The correlation with more metal rich sequences is significantly weaker but these parameter configurations viewed as a group show a high degree of internal correlation. Masses, rms residuals and $E(B-V)$ values between different model runs are tightly correlated whenever the agecorrelation is high.

\subsection{Comparison between Z2001 and PÉGASE.2}

Figure 7 also show the results from one of the PÉGASE. 2 parameter configurations. We have already noted that the different treatments of the nebular component result in very different behaviour during the first $10 \mathrm{Myr}$, and that PÉGASE. 2 cannot account for the most extreme colours. Also at later ages there are some differences between the two models. For a fixed $E(B-V)$ the qualitative agreement for ages larger than $50 \mathrm{Myr}$ is fair (Fig. 7), although PÉGASE.2 give lower ages on average. For the $E(B-V)$ free parameter cases, the pronounced peaks at $\sim 1$ and $15 \mathrm{Gyr}$ are replaced by a broader distribution for the PÉGASE. 2 model. Comparing the tracks in Fig. 5 and 4 it is not surprising that we get different results, as the PÉGASE.2 model show loops in the bvi diagram (Fig. 5) between 10 and 500 Myr and a redder colour at 1 Gyr. For both Z2001 and PÉGASE.2, a potential danger with a free fit $E(B-V)$ is that features in the evolutionary sequences, e.g. loops and kinks, may act as attractors. The degree of correlation between results from Z2001 and PÉGASE.2 with similar parameter settings is high for age and mass but smaller for $E(B-V)$ and rms residuals.

\section{Resulting constraints on the starburst physics}

In agreement with the spectroscopic analysis, we find that young objects (age < $20 \mathrm{Myr}$ ) are better reproduced by metalpoor $\left(Z_{\text {stars }} \leq 0.004\right)$ stellar components. At somewhat higher ages, a slightly higher stellar metallicity $\left(Z_{\text {stars }}=0.008\right)$ is also allowed and in some cases gives a slightly better fit. For ages on the order of $100 \mathrm{Myr}$ and above, results are inconclusive

\footnotetext{
${ }^{2}$ A Scalo98 IMF would give a factor of $\sim 2$ lower $M / L_{V}$.
}

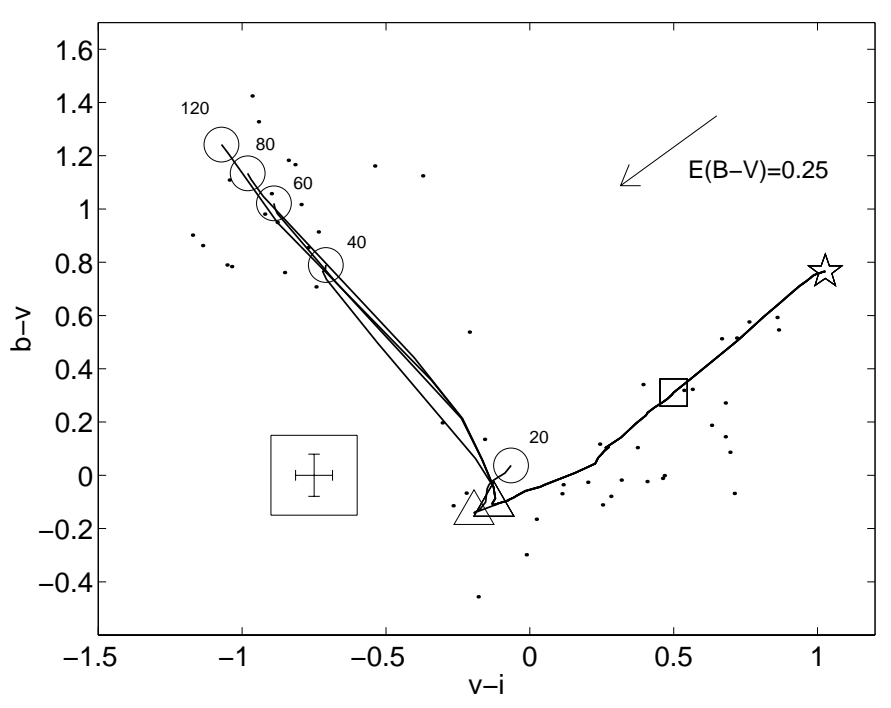

Fig. 8. Colour-colour diagram ( $v-i$ vs. $b-v)$ showing, as dots, the location of clusters with uncertainties smaller than or equal to the rectangle in the lower left. The median uncertainty is indicated by the cross. The arrow indicates how objects would be repositioned after correction for a hypothetical extinction of $E(B-V)=0.25$. Lines represent the evolutionary tracks of the models from $0.5 \mathrm{Myr}$ (circles), $10 \mathrm{Myr}$ (triangle), $1 \mathrm{Gyr}$ (square) to $15 \mathrm{Gyr}$ (Star). The predictions at the youngest age are labelled with the assumed upper mass limit. Parameters common for all scenarios: instantaneous burst, covering factor $=1.0, \alpha=2.35, M_{\text {low }}=0.08 M_{\odot}, Z_{\text {gas }}=0.002, Z_{\text {stars }}=0.001$.

regarding metallicity. Note that if a higher metallicity is adopted, much fewer objects with ages in excess of 1 Gyr are found.

We next turn our attention to the upper mass limit and slope of the IMF, which is important for regulating the ionising flux during the early stages of a burst. Hence it influences the UV-continuum and the ionised gas spectrum. The combination of red $b-v$, and blue $v-i$ colours in the colour-colour diagram at low ages in many evolutionary scenarios is a direct consequence of significant contribution from gas emission to the $v$-filter (F555W) whereas the $b$ and $i$ filters (F439W and $\mathrm{F} 814 \mathrm{~W}$ ) are free of strong lines, although nebular continuum is present in all filters. All scenarios with an upper mass limit of $M_{\text {up }}=20 M_{\odot}$ fail to reproduce the objects with red $b-v$ and blue $v-i$ colours (Fig. 8). Can we try to pin down the minimum allowed upper mass limit? A necessary requirement must be that such a scenario can reproduce the cloud of objects in the upper left part of Fig. 8. To investigate this issue, we ran a new set of evolutionary sequences with $M_{\text {up }}=40,60$, and $80 M_{\odot}$ using Z2001.

Let us first look at the Salpeter IMF. For a covering factor of 0.5 the model cannot reproduce the observations even if $M_{\text {up }}=120 M_{\odot}$. For a covering factor of 1.0, there is no problem and an upper mass limit of $80 M_{\odot}$ can be accepted (Fig. 8). However, if we increase the filling factor, we can mimic the effect of increasing $M_{\text {up }}$. Hence, even if a filling factor of unity appears unrealistic according to the current knowledge of the ISM (Kassim et al. 1989), we cannot rule out that it is higher than our generally assumed value of 0.1 . Our more conservative conclusion is therefore that for a Salpeter IMF, $M_{\text {up }} \geq 60 M_{\odot}$. 


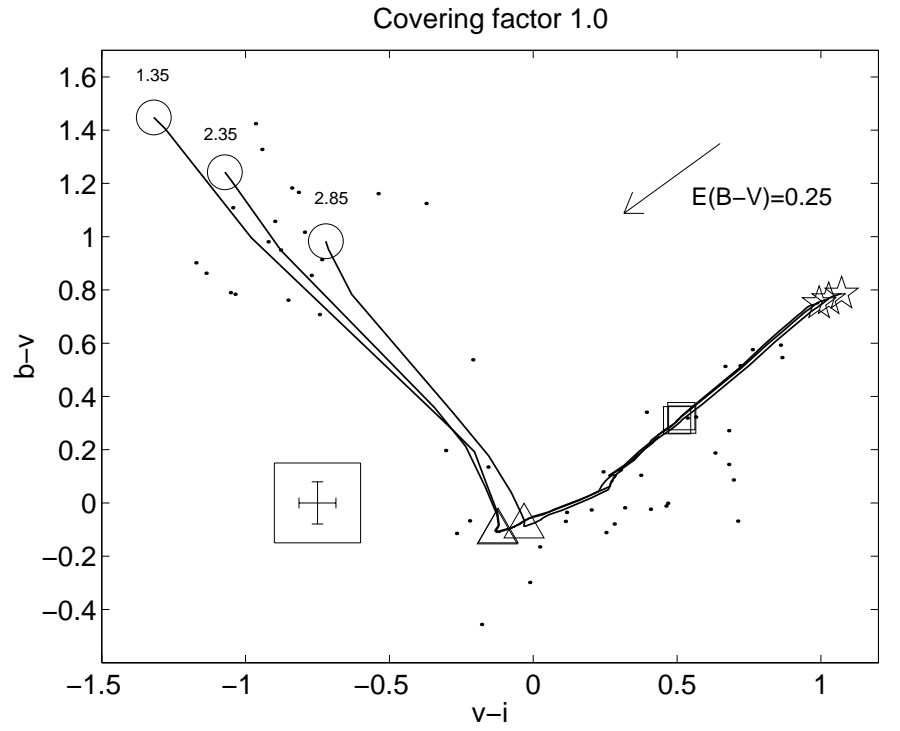

Fig. 9. Colour-colour diagram $(v-i$ vs. $b-v)$ showing the location of clusters and evolutionary scenarios with different IMF slope. Symbols have the same meaning as in Fig. 8. The scenarios are labelled with their assumed IMF slope: $\alpha=1.35,2.35$ and 2.85. Other parameters common for all scenarios: instantaneous burst $M_{\text {low }}=0.08 M_{\odot}, M_{\text {up }}=$ $120 M_{\odot}, Z_{\text {gas }}=0.002, Z_{\text {stars }}=0.001$, covering factor $=1.0$.

This is in agreement with Bergvall (1985) who from the observed $[\mathrm{OIII}]_{\lambda 5007} / \mathrm{H} \beta$ ratio found that $M_{\text {up }}>50 M_{\odot}$.

For $\alpha=1.35$, a covering factor of 1.0 give very extreme colours (Fig. 9), suggesting that cluster formation has already ceased or that $M_{\text {up }} \leq 60 M_{\odot}$. Even a covering factor of 0.5 give quite extreme colours at the earliest times, but in general offset (to bluer $v-i$ colours) from the cloud of points. This offset could possibly be explained by an average extinction of $E(B-$ $V)=0.15$, which is much more than the estimates presented in Sect. 4.1. If the sources are allowed to have a range of covering factors, an upper mass limit as low as $M_{\text {up }} \geq 40 M_{\odot}$ would provide a decent fit and the extinction may be lower. If we adopt $\alpha=1.35$ we must assume that either: $i)$ there are plenty of objects with age $10 \mathrm{Myr}$, but very few ones younger than this, or ii) $M_{\text {up }} \leq 60 M_{\odot}$, or iii) the covering factor is 0.5 and the extinction is higher than previous estimates.

For $\alpha=2.85$, a covering factor of 1.0 in combination with $M_{\text {up }}=120 M_{\odot}$ is needed to at least touch upon the the cloud of young clusters (Fig. 9). Hence we would be forced to adopt the lowest possible ages together with uniform high filling and covering factors. We regard this as unrealistic, and hence $\alpha=$ 2.85 is not our preferred IMF.

Could the IMF vary from cluster to cluster? The issue whether the IMF is universal or not has been subject to a many discussions (see Kroupa 2002). Variations could either be due to environmental or stochastical effects. The environmental effects should be similar for all clusters in ESO 338-04, but in low mass clusters the upper mass range of the IMF may be badly populated and subject to statistical noise. For a Salpeter IMF with $0.08-120 M_{\odot}$, the optical/UV emission from young clusters may be affected by stochastical effects for cluster masses below $10^{5} M_{\odot}$ (Lançon \& Mouhcine 2000), which is close to our median mass for your young clusters. Hence we cannot exclude stochastical effects among the fainter young clusters which could mimic a variable IMF slope. But in this case the average must be close to $\alpha=2.35$.

\section{Clustered vs. diffuse emission}

At first sight (Figs. 1 and 2) it appears that the star burst region is dominated by emission from compact star clusters. However from Table 3 it is clear that even the central $1 \mathrm{kpc}$ is dominated by diffuse emission. Of course, the diffuse emission is also likely to be clustered at some level and include open clusters, $\mathrm{OB}$ associations etc.

The integrated luminosity distribution of clusters in ESO 338-04 appears to have a Gaussian shape with a peak at $M_{V} \approx-10$ (ÖBR98) but this is primarily a consequence of the photometric completeness limit and a mix of ages with different $M / L$. In each age bin containing a significant number of clusters, the luminosity function (LF) is best described by a power law $\mathrm{d} N / \mathrm{d} L \propto L^{-\beta}$, with power law index $\beta=1.8 \pm 0.15$ down to the completeness limit. This is valid also for the $\mathrm{F} 218 \mathrm{~W}$ data. From this we can estimate, roughly, how large a fraction of the clusters that fall below the completeness limit. We estimate, that if we correct for clusters below the detection threshold, the clusters would make up $40 \%$ of the light in F218W. However, although the number of young clusters is small outside the central kpc, diffuse $\mathrm{H} \alpha$ emission is present out to much greater radius (Bergvall \& Östlin 2002). Is this gas ionised in situ by a more diffuse population of young stars, or by leakage of Lyman continuum photons from the central burst? The $\mathrm{H} \alpha$ emission inside $r=1.075 \mathrm{kpc}$ corresponds to $80 \%$ of the total $\mathrm{H} \alpha$ emission in ESO 338-04, which may bring the fraction of the total UV emission from the galaxy associated with young clusters, back down close to $30 \%$ if the ionisation is in situ. Hence, the fraction of $2000 \AA$ emission, and hence young stars, coming from young clusters should be in the range 30 to $40 \%$.

Meurer et al. (1995) found only $16 \%$ of the $2200 \AA$ emission in ESO 338-IG04 to come from clusters, but this was estimated in a different way (using de-convolution of the prerefurbishment HST/FOC images). The average value for nine starbursts in Meurer et al. (1995) is 20\%. We have simply integrated the emission coming from clusters (including a significant aperture correction) in all passbands and compared to the integrated emission over a circularly defined starburst area. Also our integrated starburst magnitude (see Table 3 ) is brighter by $\sim 1$ mag compared to Meurer et al. (1995). Apparently, our WFPC2 observations have been more sensitive and the fact that we get similar numbers for the other passbands, and find a monotonic increase in the cluster fraction with shorter wavelength, give us full confidence in our numbers.

Zepf et al. (2001) found that star clusters are responsible for $19 \%$ of the blue light in the the gas-rich merger NGC 3256, similar to our value of $21 \%$, but most other mergers have a smaller fraction of their optical light coming from young clusters (Östlin 2000), e.g. in the Antennae it is less than $10 \%$ (Whitmore \& Zhang 2002). 


\subsection{The ionisation energy budget}

From the Z2001 evolutionary scenarios, we have calculated for each cluster, the predicted output of Lyman continuum photons, and hence the predicted total $\mathrm{H} \alpha$ output associated with young clusters. The values we get have been scaled up with a factor $\sim 1.3$ to account for clusters fainter than the detection threshold. This correction has been applied to the estimates presented below.

The total observed $\mathrm{H} \alpha$ flux from ESO 338-04 is $\log (\mathrm{H} \alpha)=$ $41.7 \pm 0.2$ (Östlin et al. 1999) which has only been corrected for Galactic extinction, but since the internal extinction is estimated to be very small, $E(B-V) \leq 0.05$, it will serve our purpose as an estimate. If the clusters make up 30 to $40 \%$ of the present star formation, they should then produce $\log (\mathrm{H} \alpha)=$ 41.2 to $41.3( \pm 0.2)$.

A standard Salpeter metal-poor scenario with instant burst produces $\log (\mathrm{H} \alpha)=41.1$. Hence some emission is lacking, but it is within the uncertainty. A constant SFR for $10 \mathrm{Myr}$ gives 42.1 , i.e. much larger than the observed value. With a constant SFR for 5 Myr we get 41.9 and with an exponentially decaying SFR (e-folding time $3 \mathrm{Myr}$ ) we get 41.6. An IMF with $\alpha=1.35$ give similar values to $\alpha=2.35$. Hence these results support that the star formation time-scale is short, a few Myr at most.

\section{Star formation history as derived from clusters}

The formation of a massive star cluster is by necessity associated with star formation in the galaxy. In the present starburst in ESO 338-04, 30 to $40 \%$ of the newly formed stars are found in clusters. Are compact/massive star clusters formed as a natural consequence of star formation, or are special conditions, e.g. the high ISM turbulence prevailing in merging galaxies or circum-nuclear rings, required? In the former scenario, clusters form now and then as long as star formation is active. Star formation is normally a collective phenomenon, but only rarely (e.g. the Arches cluster, Serabyn et al. 1998) do we find young stars to form self-gravitating systems in our Galaxy. This could be a consequence of a low star formation rate density

The ability of young clusters to develop into globular cluster-like systems is connected to their environment: Whether the clusters will be gravitationally bound or not, depends on e.g. the ISM density, pressure and metallicity. Moreover, the ability for clusters to survive disruption depends on their environment (Meylan \& Heggie 1997).

For a given cluster age, the photometric completeness limit corresponds to a given stellar mass, producing a time dependent mass detection limit. The fraction of clusters in each age bin brighter than the limiting magnitude depends the cluster LF, a quantity which has been subject to some debate during the last years. The shape of the LF of old GCs in our Galaxy is Gaussian with a peak of $\log \left(M / M_{\odot}\right)=5.3$ and dispersion 0.5 (Fall \& Zhang 2001). On the other hand, the LF and MF of young clusters, e.g. in the Antennae (Whitmore et al. 1999) and ESO 338-04, is described by a power law without any sign of a turnover at a characteristic mass $\sim 10^{5} M_{\odot}$. As clusters age and are subject to internal and external dynamical effects, the lower part of the mass spectrum will be de-populated and the MF converted from power law to Gaussian (Fall \& Zhang 2001).
However, the initial MF may still be a power law. Whether clusters survive or not, the power-law MF measures the mass initially formed in star clusters.

If the initial cluster mass function is universal and known, it is straightforward to estimate what fraction of the clusters at a given age that fall below the detection threshold. However, in practise the mass integral for a power law with unbounded mass intervals diverges, and it is neccessary to fix the mass range, just like for the stellar IMF. Unfortunately, these quantities are not observationally known. One way would be to self consistently solve for the upper mass limit, but this only works when the MF is well populated. Moreover, this violates the assumption of a universal cluster MF and the same applies if the upper mass limit is taken as the observed maximum mass in the age bin in question. Our finally adopted method is quite crude and assumes a universal power-law initial mass function with a mass range $10^{3} M_{\odot}$ to $5 \times 10^{6} M_{\odot}$.

The time dependent correction factor for clusters below the detection treshold can now be calculated and ranges from 1.25 at low ages, where we see most of the mass (at least in systems with small reddening), to $\sim 5$ at 15 Gyr where we see only about $20 \%$. Assuming a Gaussian initial cluster mass function (e.g. to simulate that many low mass clusters get disrupted) would give similar numbers. It is not vitally important if clusters are gravitationally bound or not. Our estimate will always give a lower limit to the cluster SFR.

For each scenario, we have included the uncertainty of \pm 0.15 in the power law index $\beta$ of the LF and an uncertainty in the completeness limit of $0.25 \mathrm{mag}$. The completeness limit used is $\sim 1$ mag brighter than the photometric limit. This is to get closer to the real $100 \%$ completeness, and also removes the most uncertain objects.

\subsection{The young starburst}

Figure 10 shows the SFR vs. time during the last $100 \mathrm{Myr}$ as derived from clusters for 4 evolutionary scenarios. The patterns look close to identical. For instantaneous burts the main features are: a small peak at $0<t \leq 3 \mathrm{Myr}$, followed by a decrease for $3<t \leq 6 \mathrm{Myr}$, after which the main peak occur in the interval $6<t \leq 10 \mathrm{Myr}$. Another significant peak occurs at $20<t \leq 30$ after which the cluster SFR drops slowly but remains positive until $40 \mathrm{Myr}$.

For longer bursts (constant SFR during $10 \mathrm{Myr}$, 3rd panel in Fig. 10) the highest peak is shifted to the interval $10<t \leq$ $15 \mathrm{Myr}$, while the peak at $25 \mathrm{Myr}$ is not affected (see third panel in Fig. 10). Changing the IMF does not affect the pattern, only the scaling of the SFR.

The relatively low SFR in the first bin $(0<t \leq 3 \mathrm{Myr})$ is not a consequence of a small number of clusters, but is due to their low estimated total mass. The scarcity of objects with ages near 5 Myr can be seen also in Figs. 8 and 5: many objects are clustered around $v-i=0, b-v=0$ and $v-i=-1, b-v=1$, but few in between. Clearly, at such small ages with rapid stellar evolution, ages will be sensitive to details in the stellar models. The dip at 15 Myr could possibly be attributed to features in the multi-dimensional colour plane described by the scenarios. 


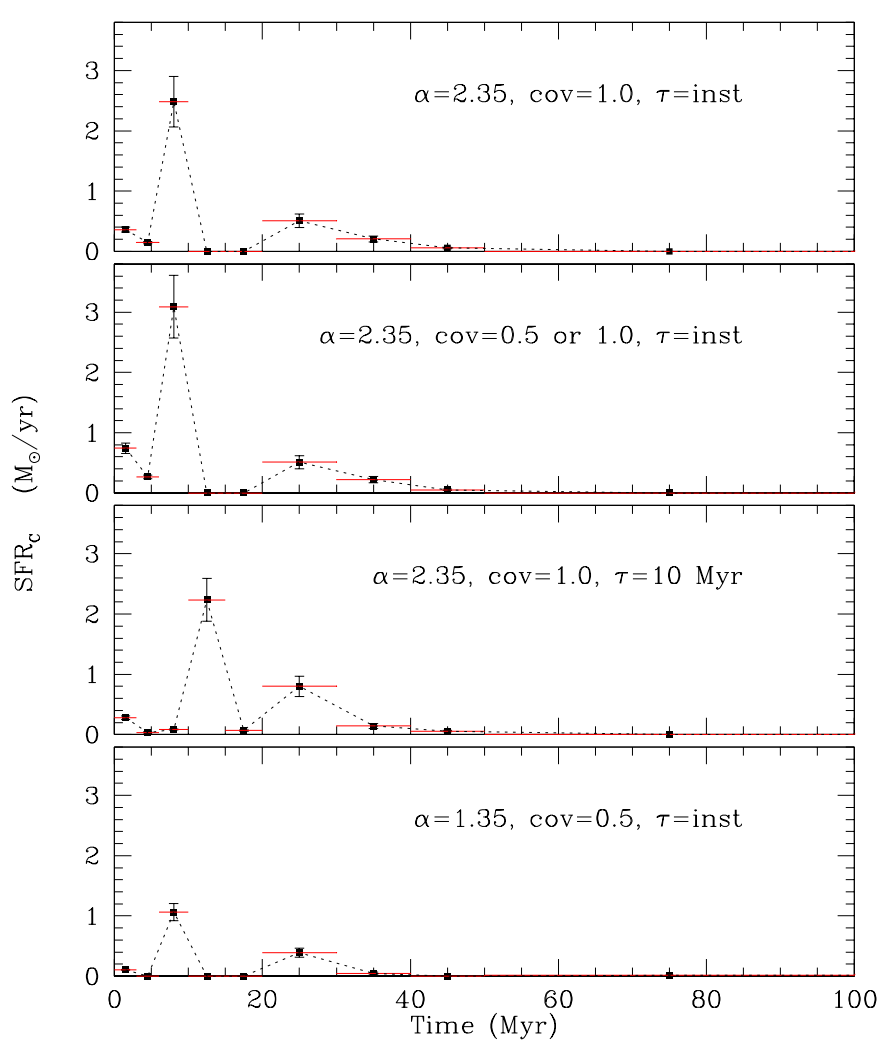

Fig. 10. The star formation rate derived from clusters as a function of time during the past $100 \mathrm{Myr}$ for 4 different evolutionary scenarios, all with $Z_{\text {gas }}=0.002, Z_{\text {stars }}=0.001$ and mass range $0.08-120 M_{\odot}$. First (top) panel: Salpeter IMF ( $\alpha=2.35)$ with instantaneous burst and covering factor $=1.0$. Second panel: Salpeter IMF with instantaneous burst when the covering factor is allowed to vary. Third panel: Salpeter IMF with a burst duration of $10 \mathrm{Myr}$ and covering factor 1.0. Fourth panel: $\alpha=1.35$ IMF with instantaneous burst and covering factor 0.5 . Error bars in the $X$-direction shows the width of the time window, and points are connected with dotted lines to guide the eye. Error bars in the $Y$-direction account for the uncertainty of \pm 0.15 in the power law index $\beta$ of the LF and an uncertainty in the completeness limit of $0.25 \mathrm{mag}$.

Whether or not the SFR went down to zero some $15 \mathrm{Myr}$ ago, the star formation activity began to rise already 40-50 Myr ago, and appears to have peaked a little less than 10 Myr ago. Hence the starburst in ESO 338-IG04 is 40-50 Myr old and still active. This estimate is based on clusters selected from their bright optical and UV emission. It is possible that a fraction of the youngest sources are hidden in dusty cocoons, that should be visible as mid-IR sources like those found in the BCG He2-10 (Vacca et al. 2002), but see discussion below.

If there is a time-lag of a few Myr in the formation of the most massive stars (see discussion above), some of the clusters in the 5-10 Myr peak would instead be very young ( $1 \mathrm{Myr})$ and the difference in SFR between the 0-5 and 5-10 Myr intervals diminished.

\subsection{The SFR viewed over a cosmological time scale}

Judging from the scenarios with a metal-poor stellar component, there is no evidence for a significantly enhanced SFR

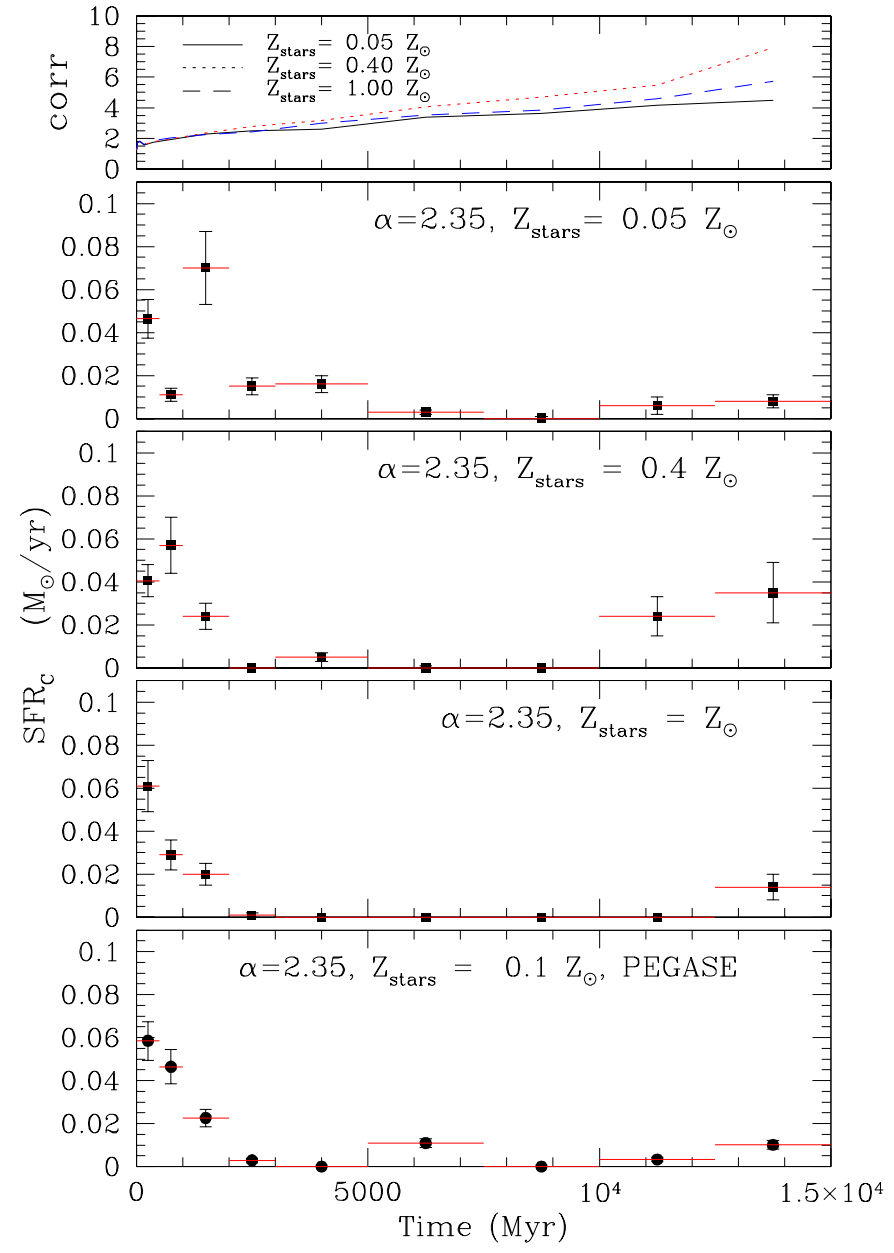

Fig. 11. The star formation rate in clusters as a function of time during the past 15 Gyr for three Z2001 evolutionary sequences with different $Z_{\text {stars }}(0.001,0.008$, and 0.02$)$, and one PÉGASE.2 sequence with $Z_{\text {stars }}=0.002$. All sequences have $\alpha=2.35$, mass range $0.08-120 M_{\odot}$ and $Z_{\text {gas }}=0.002$. The top panel shows the correction factor due to clusters below the detection limit for the different metallicities.

50-1000 Myr ago, as derived from the observed clusters. However, if we set $Z_{\text {stars }} \geq 0.008$, the cluster SFR remains high over the past Gyr although all the peaks at $t<100 \mathrm{Myr}$ remain. What happens is that many clusters are found to be younger if the metallicity is increased. Figure 11 shows the cluster SFR over the past 15 Gyr for three different metallicities $\left(Z_{\text {stars }}=0.001,0.008\right.$ and 0.020). In addition, we show the result from a PÉGASE. 2 evolutionary scenario. The present starburst is still evident in the first bin $(0<t \leq 500 \mathrm{Myr})$ but has decreased in amplitude since the SFR has been averaged over a longer time-scale. For the $Z_{\text {stars }}=0.001$ scenario, a prominent peak is found at $1.5 \mathrm{Gyr}$, and significant cluster formation occurred also between 2 and 5 Gyr ago, which was found also by ÖBR98. Assuming that all GCs older than $10 \mathrm{Gyr}$ formed during a short time scale $(\tau<1 \mathrm{Gyr})$ would produce a peak comparable to that found at $1.5 \mathrm{Gyr}$. The event 1.5 Myrs ago must have been an impressive starburst.

Bergvall \& Östlin (2002) reported that the underlying host galaxy may be significantly more metal-rich than the nebular gas. In this case one might suspect that older clusters could be 
metal-rich. For higher metallicities, the 1.5 Gyr feature remain but decrease in amplitude. The $14 \mathrm{Gyr}$ peak, on the other hand, becomes stronger. This is due to the higher $M / L$ at high ages in these scenarios, making both the mass detection limit and the masses of individual clusters higher. The unknown metallicity of older clusters and the small number of objects in each age bin presents the most important limitation for estimating the past SFR from clusters.

\subsection{The cluster mass vs. total galaxy mass}

Summing up the total cluster SFR over 15 Gyrs we get $\langle S F R\rangle=0.02 M_{\odot} / \mathrm{yr}$, or a total mass formed in clusters of $\sim 3 \times 10^{8} M_{\odot}$. The total stellar mass of this galaxy is estimated to be $\sim 4 \times 10^{9} M_{\odot}$ (Östlin et al. 2001; Bergvall \& Östlin 2002). Hence the clusters would be responsible for almost $10 \%$ of the total star formation. This is orders of magnitude higher than for most galaxies, for which the typical value is $0.3 \%$ (McLaughlin 1999, 2000). For instance, the total mass of the Milky Way globular cluster system is estimated to be on the order of $\sim 6 \times 10^{7} M_{\odot}$. As noted in Sect. 4.1, if the actual IMF is more similar to Scalo98 than Salpeter, we will overestimate the cluster masses. In addition, we count the total consumed gas mass (including the gas returned from stellar winds and explosions), but these two effects combined should not be greater than a factor of 2 . Old clusters may have suffered loss of low mass stars through dynamical evolution, but also this effect should be not greater than a factor of 2 in view of the mass to light ratios for Galactic globular clusters (Ashman \& Zepf 1998). Taking also the mass of gas $M(\mathrm{HI})=3 \times 10^{9} M_{\odot}$ into account we are still an order of magnitude above $0.3 \%$. If we count only the actually detected clusters we still get a total cluster mass of $9 \times 10^{7} M_{\odot}$ for $Z_{\text {stars }}=0.001\left(\geq 7 \times 10^{7}\right.$ for any $\left.Z_{\text {stars }}\right)$, or about one third of the total implied cluster mass. This would still give a mass fraction $\gg 0.3 \%$ if accounting for the possible systematic effects mentioned above.

The conclusion that this galaxy is, and has been over a cosmological time-scale, a very efficient cluster former is unavoidable. The open question is why? Tashiro \& Nishi (2000) suggest that the formation of gravitationally bound clusters may be favoured in low metallicity environments.

Bergvall \& Östlin (2002) found that the stellar metallicity of the host galaxy is higher than that of the ISM, which could be explained if a metal-poor gas-rich dwarf galaxy or an H I cloud has been accreted. They speculate that the unusually high metallicity, in view of the low luminosity, arise because the galaxy is rich in dark matter (see Sect 9.1), which has enabled it to retain all its metals. If this is true, the total mass fraction (including dark matter) of globular and akin clusters may not be higher than in other galaxies. Another possibility if one postulates a universal cluster formation efficiency is that for some reason the survival chances are greater in ESO 338-IG04, but then this should be a general trend among dwarf galaxies.

\section{Spatial propagation of the starburst}

The distribution of young clusters can be used to investigate if propagating star formation have occurred. In Fig. 12 we show the spatial age pattern for young clusters for a standard metalpoor Salpeter scenario (our favoured one for young objects) with a varying covering factor. There are indeed some striking patterns present in this image.

The youngest objects ( $<3 \mathrm{Myr}$ ), coded in cyan, tend to prefer a mix of covering factors. They are preferentially found in a ring like structure centred on $X=270, Y=305$ (capital $X$ and $Y$ refer to Planetary Camera pixel values, each pixel has a size of 8.3 pc, see Fig. 12) and in a group near $X=320$, $Y=400$. The former structure have in general a best fitting covering factor of 1.0 , and the latter 0.5 .

Slightly older objects ( $3 \leq$ Age $<8 \mathrm{Myr}$, coded in blue), are found in the latter group and also near the starburst centre $(X=$ $290, Y=340$ ). Objects with ages 8 to $30 \mathrm{Myr}$ are found in the centre and in a group near $(X=300, Y=260)$. Older objects (30-100) Myr are not strongly clustered, but are preferentially found in the upper half.

The ring-like structure of very young ( $<3 \mathrm{Myr}$ ) objects have a major axis diameter of $0.8 \mathrm{kpc}$. A velocity of $1 \mathrm{~km} \mathrm{~s}^{-1}$ correspond to $1 \mathrm{pc} / \mathrm{Myr}$. The $\mathrm{H} \alpha$ velocity dispersion is $\sigma_{\mathrm{H} \alpha}=$ $43 \mathrm{~km} \mathrm{~s}^{-1}$ (Östlin et al. 2001) implying a turbulent half ring crossing time of $10 \mathrm{Myr}$. Hence the coeval formation of this ring can not have been triggered by a wave travelling at the ISM turbulent velocity, but perhaps by a supernovae driven wind. The grouping of very young objects in the upper part is more compact and consistent with a coeval formation if random motions of $0.1 \mathrm{kpc}$ are considered. To the right of this grouping several diffuse emission-line regions without compact clusters are present (see Fig. 1), which may possibly be the youngest sites of star formation. The $\sim 10 \mathrm{Myr}$ (yellow) grouping in the lower part $(X=300, Y=260)$ has a similar dimension. Their age is consistent with a localised formation followed by diffusion by random motions. In this region we find an interesting boundary between $\sim 10$ and $\sim 25$ Myr old objects, although one can not exclude that a dust filament is responsible for the apparent ridge of $\sim 25 \mathrm{Myr}$ objects. The centre $(X=290, Y=340)$ is close to the centre of the outer isophotes and could therefore represent the central potential of the galaxy. Here cluster formation appears to have been continuing over the last 40 Myr. The present starburst may have started in the region slightly west (up in Fig. 12) of the centre where almost all of the sources older than $30 \mathrm{Myr}$ are located. A possible scenario is that the upper and lower 8 to $30 \mathrm{Myr}$ groups represent approaching complexes that have triggered the young shell-like structure. Indeed, the $\mathrm{H} \alpha$ velocity field show that the eastern part of the galaxy (down-left in Fig. 12) may be falling in towards the centre (Östlin et al. 2001). Moreover, the $\mathrm{H} \alpha$ velocity dispersion is higher east of this region $\left(\sigma_{\mathrm{H} \alpha}=70 \mathrm{~km} \mathrm{~s}^{-1}\right)$ and there are suggestions of a perpendicular velocity component. Further out towards east (outside the Planetary Camera FOV shown in Fig. 1) we find some diffuse emission-line regions and clusters ${ }^{3}$.

Although projection effects and the velocities of individual clusters are not known, our results provide evidence for

\footnotetext{
3 Some blueish clusters in this tail (Östlin et al. 2001; Bergvall \& Östlin 2002) are visible in the Wide Field chip 3, but their photometry is too uncertain to assess their ages.
} 


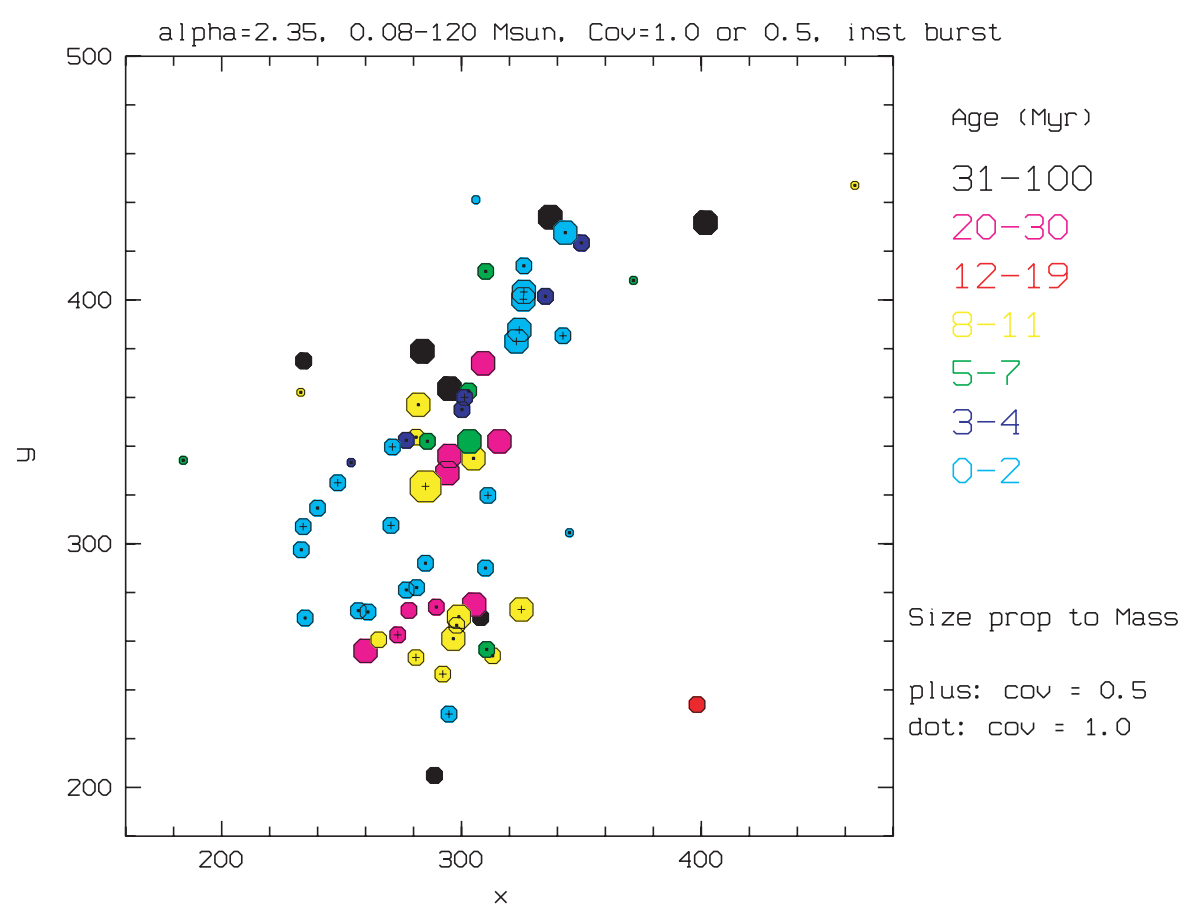

Fig. 12. Spatial distribution of young clusters using best fitting $E(B-V)$ and Salpeter IMF with mass range 0.08 to $120 M_{\odot}$, and metallicity $Z_{\text {stars }}=0.001$ and $Z_{\text {gas }}=0.002$. The covering factor was allowed to vary between 0.5 (+ signs) and 1.0 (dots). The size of each symbol is proportional to the logarithm of the mass of a cluster and the colour depends on the age. The $x$ - and $y$-coordinates correspond to pixel numbers in the Planetary Camera and the region showed is $14.6^{\prime \prime} \times 14.6^{\prime \prime}$, or $2.6 \times 2.6 \mathrm{kpc}$.

the spatial propagation of the starburst over the last $30 \mathrm{Myr}$. Supersonic triggering appears to be necessary to explain some of the youngest features.

The star formation pattern and the conclusions remain unaffected if a fixed $E(B-V)=0.0$ or 0.05 , or a fixed covering factor, is adopted. The same applies to changing the IMF: ages would change slightly, but not the pattern. If massive star formation would be associated with a time-lag, as discussed above, most features would remain, e.g. the young ring, but it would be a few Myrs older, but still too young for a sound wave to have triggered their formation. Some of the $\sim 10$ Myr objects (in yellow) would now rather be just a few Myr.

\subsection{Spatial distribution of old globular clusters}

For old ( $\geq 0.8 \mathrm{Gyr}$ ) clusters the time-scale is too long for the position of individual clusters to have any correspondence with their place of birth. Instead, we examine the spatial distribution of the ensemble of old clusters (Fig. 13). Intermediate age $(0.8 \leq$ age $<5$ Gyr) clusters (open shaded symbols in Fig. 13) have a flattened distribution with minor to major axis ratio $b / a \approx 0.5$, with the same position angle as the galaxy as a whole. The isophotes of ESO 338-04 have an axis ratio $b / a=0.48$ (Bergvall \& Östlin 2002). The colours of the host galaxy at radii sufficiently large that the starburst contribution is negligible, indicate an age in the range 2-8 Gyr and a relatively high stellar metallicity. That the intermediate age globular cluster system has an identical flattening suggests a common origin or, if the lower age of the clusters is taken at face value, that these formed in the existing gravitational potential of the host galaxy. If a higher metallicity is adopted for the clusters, the age difference would increase.

On the contrary, clusters older than $5 \mathrm{Gyr}$ (filled black symbols in Fig. 13) show no apparent flattening $(b / a \approx 1.0)$ suggesting the presence of an old spherical halo, and that they are indeed metal-poor. The number of objects is too small to study the radial distribution in detail, but the effective radius (containing half the number of $>5$ Gyr clusters) is $\sim 1.4 \mathrm{kpc}$. If a Gaussian LF similar to the one for Galactic GCs $\left(M_{v}^{\text {peak }}=\right.$ $-7.2, \sigma=1.3 \mathrm{mag}$ ) is assumed, the total number (including non detected ones) of old halo clusters should be $\sim 50$. For a typical GC richness of spherical systems $(T=5$, see Ashman \& Zepf 1998 for a definition of $T$ ) 50 GCs would then imply a mass of the halo of $10^{10} M_{\odot}$. This thought experiment supports the idea of a dark matter-rich halo discussed in Sect. 8.3

\section{Discussion - star formation and dusty molecular clouds}

Star formation in our Galaxy and elsewhere is associated with dusty giant molecular clouds. Massive starbursts like M 82 and Arp 220 are reddened by several tens of magnitudes of optical extinction (Genzel et al. 1998), and a large fraction of the UV power is absorbed and re-radiated in the infrared. However, the extinction inferred from young star clusters in M 82 is only of the order of $A_{V}=1$ (de Grijs et al. 2001) compared to the 


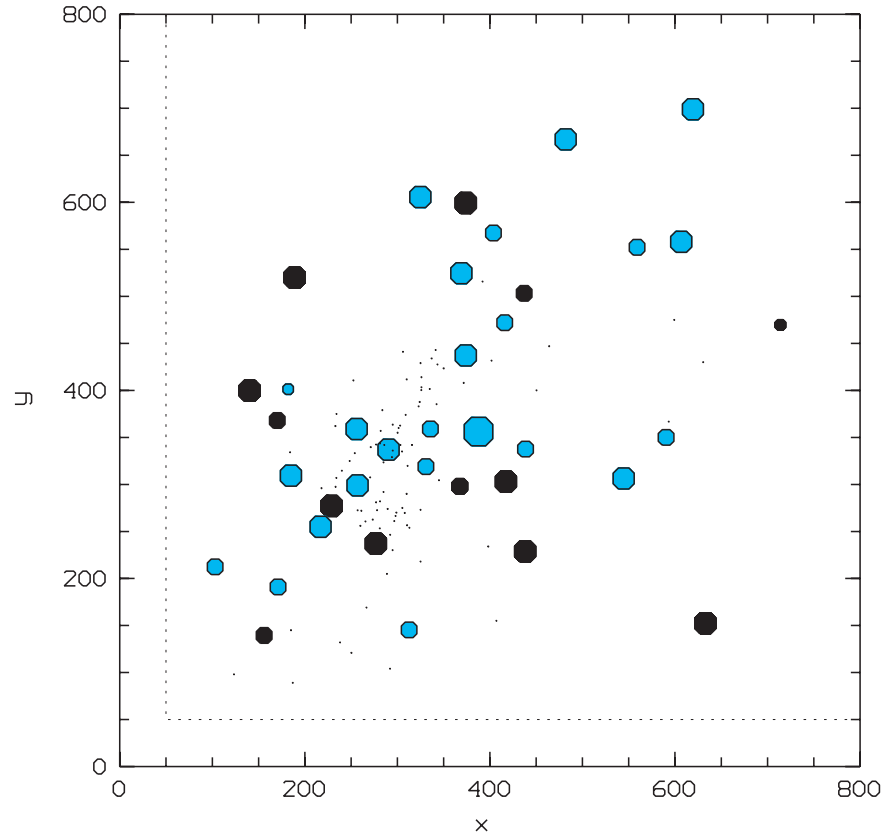

Fig. 13. Spatial distribution of old (age $>5 \mathrm{Gyr}$, filled black symbols) and intermediate age $(0.8<$ age $\leq 5 \mathrm{Gyr}$, open shaded symbols $)$ clusters with size proportional to mass. The small dots younger ( $\leq 0.8 \mathrm{Gyr})$ objects. The axes show the spatial coordinates, in units of PC pixels. The region delimited by a dashed line $(X<50, Y<50)$ lies at the border to the wide field camera CCDs and is subject to vignetting, and therefore not included in our analysis.

50 mag derived from IR data (Förster Schreiber et al. 2001). In the Antennae, the youngest clusters have high extinction $A_{V}=3$ to 5 (Zhang et al 2001), and are spatially correlated with the IR emission, whereas the nuclei have $A_{V} \sim 50$. Obviously, optical selection systematically picks out sources with lower than average extinction.

The situation in ESO 338-04 is quite different. Looking at Figs. 4 and 5 we see no tendency for the youngest objects to be reddened. A few sources lie to the upper right compared to the locus of the evolutionary sequences at the lowest age, as if they were reddened by $E(B-V) \leq 0.25$. However, there are more sources to the down-left, and the observations are consistent with a dust free population plus random effects (integer number of massive stars, and small variations is gas filling and covering factors) plus photometric errors. Although our sample is biased towards optically bright clusters it is nevertheless surprising that we do not see any young sources just about to come out of their dusty cocoons in which they, according to common wisdom, should have formed. Note that there are nebular emission dominated regions without associated point sources west of the centre, which may represent young objects, still partly embedded.

The lifetime of Galactic giant molecular clouds against photo- dissociation and ionisation is on the order of $30 \mathrm{Myr}$ once massive star formation commence (Williams \& McKee 1997). If we insist that star formation is associated with dusty molecular clouds, then the lack of young reddened sources suggest that in metal-poor starbursts with high ISM turbulence
(Östlin et al. 2001) and high star formation efficiency, this time scale is much shorter. ESO 338-04 is a hot IRAS source $\left(S_{60} / S_{100}=1.7\right)$ but the FIR luminosity is modest $L_{\mathrm{IR}}=$ $5.8 \times 10^{9} L_{\odot}$ and the IR to blue luminosity, $L_{\mathrm{IR}} / L_{B}=1.0$ (solar units), is very low with respect to its $f_{60} / f_{100}$ ratio (Bergvall et al. 2000; Dulzin-Hacyan et al. 1988). The derived dust mass is only of the order of $10^{5} M_{\odot}$ (see also Calzetti et al 1995). This implies small amounts of dust heated to high temperatures, and fits well with the short time-scale scenario.

A short time-scale for molecular cloud destruction would be consistent with the difficulty to find $\mathrm{CO}$ emission in metalpoor dwarf starbursts (Kunth \& Östlin 2000) since not only the $\mathrm{CO}$ to $\mathrm{H}_{2}$ conversion factor may be low, but the gas would stay in molecular form a shorter time. For ESO 338-04, $L_{\mathrm{CO}(1-0)}<$ $7 \times 10^{27} \mathrm{~W}$, so $\log \left(L_{\mathrm{CO}} / L_{\mathrm{IR}}\right)<-8.4$ (Bergvall, unpublished), implying $M_{\mathrm{H}_{2}} \leq 7 \times 10^{6} M_{\odot}$ if a standard $\mathrm{CO}$ to $\mathrm{H}_{2}$ conversion factor is used. As the mass of young ( $\leq 10 \mathrm{Myr}$ ) stars (in clusters and diffuse) is $\sim 10^{7} M_{\odot}$, and star formation efficiency must be well below $100 \%$, the molecular gas dissociation time scale should be a few Myr at most.

While we see several very young ( $\sim 1$ Myr) clusters, Fig. 10 shows that the global star formation intensity may have passed its peak. This combined with the tendency to pick out sources with low extinction suggest that some star formation could be hidden after all. Of course we may happen to observe this galaxy just as the last reservoirs of dusty molecular gas are depleted, but we would still need to postulate a short dust destruction time scale.

If the time scale for molecular cloud destruction is unusually short, it is possible that a young cluster would still not have reached its peak UV luminosity when becoming naked. This could give us an additional selection effect to account for the lack of young reddened clusters.

The cluster richness implies a high cluster and star formation efficiency (see Sect. 8.3), which could be a natural cause for the short dust destruction time-scales since the UV radiation density in the ISM is high. It is surprising that the star formation efficiency can be so high in a galaxy with a metal-poor ISM and little dust.

\section{Conclusions}

Using multicolour photometry from 2000 to $8000 \AA$, we have analysed the temporal and spatial evolution of star clusters and the starburst in the Luminous Blue Compact Galaxy ESO 33804. Our main results are:

- Using an extensive set of spectral evolutionary synthesis scenarios and Monte-Sarlo simulations we conclude that the overall age distribution found is very robust. Massive star clusters with ages from 1 Myr up to the maximum age considered in the models ( $15 \mathrm{Gyr}$ ) are present in this galaxy.

- A careful treatment of the nebular emission is crucial for modelling the properties of young star clusters.

- In accordance with previous studies, we find the starburst in ESO 338-04 to be almost unaffected by dust extinction. There is no evidence for a large population of young 
sources about to come out of their dusty cocoons. We speculate that this can be explained by a short time-scale, $\leq 1 \mathrm{Myr}$, for destruction of dusty molecular clouds.

- The cluster formation history have been used to constrain the overall star formation history in this galaxy. The present starburst began 40-50 Myr ago and is still active. There are indications that the SFR has varied during time, and that it has already passed its peak.

- Young clusters are responsible for 30 to $40 \%$ of the star formation and the starburst luminosity output in the UV, which is a high number, even for starbursts.

- Seen over a cosmological time, this galaxy has been a remarkably efficient cluster former. The reason for this remains an enigma. The intermediate age population has a flattened distribution with the same flattening as the host galaxy. The old clusters have a spherical distribution.

- The observations are well reproduced by a Salpeter IMF ( $\alpha=2.35$ ) with an upper mass limit $M_{\text {up }} \geq 60 M_{\odot}$. A steeper IMF ( $\alpha=2.85)$ cannot reproduce the data. A flatter IMF ( $\alpha=1.35$ ) cannot be excluded but requires additional constraints to provide a good fit. An ISM metallicity close to the spectroscopic value is strongly favoured. Our results suggest that the formation time-scale for an individual cluster is at maximum a few Myrs.

- We find evidence for spatially correlated formation of groups of clusters, some requiring supersonic triggering velocities, e.g. from supernovae winds.

Acknowledgements. This work was supported by the Swedish Research Council and the Swedish National Space Board.

\section{References}

Ashman, K. M., \& Zepf, S. E. 1998, Globular Cluster Systems (Cambridge University Press)

Bergvall, N. 1985, A\&A, 146, 269

Bergvall, N., \& Östlin, G. 2002, A\&A, 390, 891

Bergvall, N., Masegosa, J., Östlin, G., \& Cernicharo, J. 2000, A\&A, 359,41

Biretta, Lubin., et al. 2002, WFPC2 Instrument Handbook, Version 7.0 (Baltimore: STScI).

Brodie, J. P., \& Huchra, J. P. 1991, ApJ, 379, 157

Buat, V., Burgarella, D., Deharveng, J. M., \& Kunth, D. 2002, A\&A, 393, 33

Calzetti, D., Kinney, A. L., \& Storchi-Bergmann, T. 1994, ApJ, 429, 582

Calzetti, D., Bohlin, R. C., Kinney, A. L., Storchi-Bergmann, T., \& Heckman, T. 1995, ApJ, 443, 136

Charlot, S., Worthey, G., \& Bressan, A. 1996, ApJ, 457, 625

Clegg, R. E. S, \& Middlemass, D. 1987, MNRAS, 228, 759

De Grijs, R., O'Connel, R. W., \& Gallagher, J. S. 2001, AJ, 121, 768

Dulzin-Hacyan, D., Moles, M., \& Masegosa, J. 1988, A\&A, 206, 95

Fall, S. M., \& Zhang, Q. 2001, ApJ, 561, 751

Ferland, G. J. 1996, Hazy, a Brief Introduction to Cloudy. University of Kentucky Department of Physics and Astronomy Internal Report

Förster Schreiber, N. M., Genzel, R., Lutz, D., Kunze, D., \& Sternberg, A. 2001, ApJ, 552, 544

Fioc, M., \& Rocca-Volmerange, B. 1999 [astro-ph/9912179]
Genzel, R., Lutz, D., et al. 1998, ApJ, 498, 579

Guzmán, R., Gallego, J., Koo, D. C., et al. 1997, ApJ, 489, 559

Hopkins, A. M., Schulte-Ladbeck, R. E., \& Drozdovsky, I. O. 2002, AJ, 124, 862

Iye, M., Ulrich, M. -H., \& Peimbert, M. 1987, A\&A, 186, 84

Kassim, N. E., Weiler, K. W., Erickson, W. C., \& Wilson, T. L. 1989, ApJ, 338, 152

Kennicutt, R. C. 1998, in The Stellar Initial Mass Function, ed. G. Gilmore, \& D. Howell, ASP Conf. Ser., 142, 1

Kroupa, P. 2002, Science, 295, 82

Kunth, D., \& Östlin, G. 2000, A\&ARv, 10, 1

Lançon, A., \& Mouhcine, M. 2000, in Massive Stellar Clusters, ed. A. Lançon, \& C. Boily, ASP Conf. Ser., 211, 63

Le Fèvre, O., Abraham, R., Lilly, S. J., et al. 2000, MNRAS, 311, 565

Leitherer, C. 1998, in The Stellar Initial Mass Function, ed. G. Gilmore, \& D. Howell, ASP Conf. Ser., 142, 61

Lejeune, T., Cuisinier, F., \& Buser, R. 1998, A\&AS, 130, 65L

Lilly, S. J., Le Fèvre, O., Hammer, F., \& Crampton, D. 1996, ApJ, 460, L1

Madau, P., Ferguson, H. C., Dickinson, M. E., et al. 1996, MNRAS, 283,1388

Maíz-Appelániz, J. 2001, ApJ, 563, 151

Mas-Hesse, J. M., \& Kunth, D. 1999, A\&A, 349, 765

Massey, P., \& Hunter, D. A. 1998, ApJ, 493, 180

McKee, C. F., \& Tan, J. C. 2002, Nature, 416, 59

McLaughlin, D. 2000, in Massive Stellar Clusters, ed. A. Lançon, \& C. Boily, ASP Conf. Ser., 211, 63

McLaughlin, D. 1999, AJ, 117, 2398

Meurer, G. R., Heckman, T. M., Leitherer, C., et al. 1995, AJ, 110, 2665

Meurer, G. R., Heckman, T. M., \& Calzetti, D., ApJ, 521, 64

Meylan, G., \& Heggie, D. C. 1997, A\&ARv, 8, 1

Miller, G., \& Scalo, J. 1982 ApJ, 263, 259

Östlin, G., Bergvall, N., \& Rönnback, J. 1998, A\&A, 335, 85 (ÖBR98)

Östlin, G. 2000, in Massive Stellar Clusters, ed. A. Lançon and C. Boily, ASP Conf. Ser., 211, 63

Östlin, G., Amram, P., Bergvall, N., et al. 2001, A\&A, 374, 800

Pandey, A. K., Ogura, K., \& Sekiguchi, K. 2000, PASJ, 52, 847

Pascarelle, S. M., Windhorst, R. A., \& Keel, W. C. 1998, AJ, 116, 2659

Raimann, D., Storchi-Bergmann, T., Bica, E., Melnick, J., \& Schmitt, H. 2000, MNRAS, 316, 559

Salpeter, E. 1955, ApJ, 121, 161

Scalo, J. M. 1998, in The Stellar Initial Mass Function, ed. G. Gilmore, \& D. Howell, ASP Conf. Ser., 142, 201 (Scalo98)

Schulte-Ladbeck, R. E., Hopp, U., Greggio, L., Crone, M. M., \& Drozdovsky, I.O. 2001, AJ, 121, 3007

Searle, L., \& Sargent, W. L. W. 1972, ApJ, 173, 25

Seaton, M. J. 1979, MNRAS, 187, 73

Serabyn, E., Shupe, D., \& Figer, D. F. 1998, Nature, 394, 448

Tashiro, M., \& Nishi, R. 2000, ApJ, 536, 277

Vacca, W. D., Johnson, K. E., \& Conti, P. S. 2002, AJ, 123, 772

Whitmore, B. C., in A Decade of Hubble Space Telescope Science Eds. Livio M., Noll K., Stiavelli M. (Cambridge University Press), in press [astro-ph/0012546]

Whitmore, B. C., \& Zhang, Q. 2002, AJ, 124, 1418

Whitmore, B. C., Zhang, Q., Leitherer, C., et al. 1999, AJ, 118, 1551

Williams, R. E., Blacker, B., Dickinson, M., et al. 1996, AJ, 112, 1335

Williams, J. P., \& McKee, C. F. 1997, ApJ, 476, 166

Zackrisson, E., Bergvall, N., Olofsson, K., \& Siebert, A. 2001, A\&A, 375,814

Zepf, S. E., Ashman, K. M., English, J., Freeman, K. C., \& Sharples, R. M. 1999, AJ, 118, 752

Zhang, Q., Fall, S. M., \& Whitmore, B. C. 2001, ApJ, 561, 727 